\title{
ANÁLISIS CRíTICO DEL PLAN DE SAUVEGARDE ET DE MISE EN VALEUR DEL BARRIO PARISINO DEL MARAIS A TRAVÉS DE SU EVOLUCIÓN ${ }^{1}$
}

\author{
A CRITICAL ANALYSIS PLAN DE SAUVEGARDE ET DE MISE EN VALEUR OF THE PARISIAN QUARTER \\ THE MARAIS THROUGH EVOLUTION
}

\section{ANDRÉ DEL² / ANGIE SHIMABUKURO SHIMABUKURO³}

Fecha de recepcíñ: 28 de junio de 2014.

Fecha de aprobación: 28 de setiembre de 2014.

\begin{abstract}
RESUMEN
El presente artículo ha sido redactado a partir de un trabajo de investigación desarrollado en unos estudios de maestría. Francia posee zonas urbanas protegidas denominadas secteur sauvegardé. La posibilidad de crear y delimitar dichas zonas fue instituida a partir de la promulgación de la Ley Malraux, en 1962. Dichas zonas son sometidas a reglas particulares en función a un Plan de Salvaguardia y de Puesta en Valor (PSMV). La investigación realizada muestra la evolución de los proyectos del PSMV del Marais a partir de 1969 hasta el 2013. El análisis pone en evidencia el resultado de un proceso lento de definición y de adaptaciones sucesivas a los reglamentos implementados. Hay un constante desfase entre los objetivos teórico-práctico definidos desde el punto de vista de la reglamentación y de la aplicación.
\end{abstract}

\section{PALABRAS CLAVE}

Secteur sauvegardé *, puesta en valor, patrimonio, Marais.

\section{ABSTRACT}

This article has been written based from a Master's research. France has an urban area protected that is referred to as secteur sauvegardé. The possibility to create and limit the zones was instituted after the promulgation of Malraux Law, in 1962. These zones are subdued to special rules according to the PSMV. The research shows the evolution of the Marais PSMV projects, from 1969 to 2013. Analysis reveals a slow process of results for definitions and successive adaptations of the rules implemented. There is a continued gap between theoretical and practical objectives defined from regulation and application point of view.

\section{KEYWORDS}

Secteur sauvegardé, improvement, heritage, Marais.

\footnotetext{
1. El presente artículo se basa en un trabajo de investigación sustentado en París, el 18 de junio del 2013, con calificación de Excelencia en la Maestría Historia y Civilización comparada, especialidad en Ciudad, Arquitectura y Patrimonio de la Universidad Paris 7 Diderot y ENSAPVS con la dirección de André Del y Philippe Rivoirard.

2. Ingeniero de Trabajos Públicos del Estado. Director del laboratorio Espace Virtuel de Conception Architecturale et Urbaine (EVCAU), Ecole nationale supérieure d'architecture Paris Val de Seine (ENSAPVS). Sus ejes de investigación son la utilización y desarrollo de tecnologías digitales para la ciencia de la historia y la modelización estructurada de conjuntos patrimoniales, aplicación del SIG geo-histórico. (andre. del@evcau.archi.fr).

3. Doctoranda en Historia y Civilizaciones de la Université Paris 7 Diderot - École Doctorale 382 - Laboratoire Identités, Cultures, Territoires EA 337 con el tema de investigación Préservation du Patrimoine de Barrios Altos à Lima con la dirección de Anna Caiozzo. Ha realizado una maestría de investigación en Histoire et Civilisation comparées, spécialité Ville, Architecture et Patrimoine en la Université Paris 7 Diderot \& ENSAPVS y una maestría de investigación en Histoire de l'Art en la Université de Besançon. Arquitecta de la Facultad de Arquitectura, Urbanismo y Artes de la Universidad Nacional de Ingeniería, Lima-Perú. (angieshimabukuro@gmail.com).
} 


\section{Introducción: contexto local y jurídico}

París, por la densidad de su patrimonio monumental, ha sido beneficiada por las leyes de protección del patrimonio. Existe dos sectores de salvaguardia parisinos: el Marais, barrio histórico, y una gran parte del séptimo arrondissement. ${ }^{1}$ Estos dos han sido beneficiados de una protección específica regida por un Plan de Salvaguardia y de Puesta en Valor $(\mathrm{PSMV})^{2}$. El Marais, barrio histórico situado entre el tercer y cuarto arrondissement de París, ha sido delimitado al oeste por la calle Beaubourg, al este por el boulevard Beaumarchais, al norte por la calle de Bretagne y al sur por el Sena. (figura 1 - Mapa del Sector de Salvaguardia del Marais.) En 1964 -luego de la ley Malraux de 1962- se creó un secteur sauvegardé, sector protegido o salvaguardado, de 126 hectáreas que cubría el conjunto del barrio del Marais el más vasto de Francia. El Marais posee un patrimonio excepcional y una gran homogeneidad tanto arquitectural como urbana. Las redes viales se encuentran fijas antes de fines del siglo XIII, ${ }^{3}$ los ejes comerciales ${ }^{4}$ de la ciudad conectan los lugares de intercambio que facilitan los desplazamientos y la concentración de las actividades económicas. A continuación, explicaremos el proceso de formación histórico del barrio.

\section{Situación histórica del Marais}

El recinto de Philippe Auguste, ${ }^{5}$ construido entre 1190 y 1210 para proteger París, incluía ya la parte del Marais. En 1240, la orden del Temple, ${ }^{6}$ entonces bien rico y poderoso, construyó un priorato rodeado de murallas al exterior del recinto de Philippe Autuste. A inicios de siglo XIII, las primeras comunidades religiosas se instalan en el Marais. Los reyes franceses solicitaban a las órdenes religiosas no construir más nuevas iglesias y capillas en la Isla de la Cité y lo realizan en el Marais.En el siglo XIV, el Marais comienza

1. División administrativa que equivale a distrito.

2. Plan de Sauvegarde et de Mise en Valeur.

3. "En 1705 , le réseau viaire est presque identique à celui de 1292 et en 1830 le plan de Paris n'offre que des variations infimes, produites par quelques transformations très locales (création de marché par exemple)" (Paris Projet, 1970, p.52 - p.53).

4. Estos ejes corresponden a las actuales calles Saint Martin, du Temple, Vieille du Temple et Saint Antoine.

5. Philippe Auguste o Philippe II (1165-1223). Su reinado supuso un periodo de mejoramiento de París. En 1187, inició la construcción de un muro del recinto sobre la rive droite (río derecho).

6. Orden militar cristiana de la Edad Media. a devenir en un barrio para nobles. El rey Charles $\mathrm{V}^{7}$ hizo construir un nuevo recinto que estuvo anexado a la ciudad y que incluía todo el Marais. Durante la guerra de cien años ${ }^{8}$, Charles $\mathrm{V}$ debía abandonar la estadía en el Palais de la Cité y encuentra una nueva residencia en el hotel Royal des Tournelles, ${ }^{9}$ el cual queda como residencia parisina de los reyes franceses durante ciento cincuenta años hasta la muerte del rey Henri II. ${ }^{10}$ Poco a poco, a partir de 1358 , comienza a instalarse la nobleza y la clase más rica que edificó sus residencias al lado de la del rey. Del siglo XVI al XVII, se produjo el apogeo del barrio; así, la densidad de las habitaciones burguesas aumentó ${ }^{11} \mathrm{y}$ la Plaza Royale ${ }^{12}$-actual Plaza de Vosges- deviene en el centro elegante de París. ${ }^{13}$ Durante el apogeo del Marais, aparecen también nuevas comunidades religiosas. ${ }^{14}$

A partir de inicios del siglo XVIII, la nobleza comienza paulatinamente a abandonar el Marais y se muda hacia el oeste y los artesanos reemplazan a la nobleza. Luego de la era de mundanería que carece la época de urbanización del Marais de Henri IV, ${ }^{15}$ el barrio se vuelve austero. El Marais, entonces, deviene en un "viejo barrio" y comienza una era de saturación y de

7. Charles V Charles le Sage (Carlos el Sabio) Fue rey de Francia de 1364 a 1380.

8. Conflicto entre los reinos de Francia e Inglaterra de 1337 a 1453.

9. Construido en 1417 y situado donde se encuentra la Place de Vosges.

10. Henri II (1519-1559). Fue rey de Francia de 1547 a 1559.

11. El hotel Carnavalet (1544), el hotel Lamoignon (1585), el hotel de Mayenne (1613), el hotel Sully (1624), y el hotel Salé (1656). La última realización prestigiosa del Marais fue la del hotel Rohan-Soubise (1705) para el Duque de Rohan. (Bonnermeier, p.12p.13).

12. La plaza fue inaugurada en 1612 con motivo del matrimonio de Louis XIII con Anne d'Autriche. En el pabellón Nº vivió Victor Hugo, entre 1832 y 1848.

13. Luego de la muerte de Henri II, la reina Catherine de Médicis renuncia a vivir en Tournelles y manda a construir el Palais des Tuileries (Palacio de Tullerías). Sin embargo, el Marais queda como un barrio aristocrático. Ella luego decide demoler el hotel Royal des Tournelles. El rey Henri VI retoma entonces la idea de Charles IX (Carlos IX) ordenando en 1605 el acondicionamiento de una gran plaza construida de cuatro lados de una misma simetría. " L'ensemble de la Place Royale fait à la fois impression élégante et pratique, harmonieuse et claire. Elle devenait au XVIlème siècle le centre du Paris élégant, représentant une sorte de cœur du Marais" (Bonnermeier, p.11).

14. Les Minimes, les Capucines (1524), les Filles de Saint-Elisabeth (1626) et les Jésuites.

15. Henri IV (1553-1610) fue rey de Navarre de 1572 a 1610; luego, rey de Francia de 1589 a 1610. Fue el primer soberano francés de la rama de Bourbon, de la dinastía capétienne. 


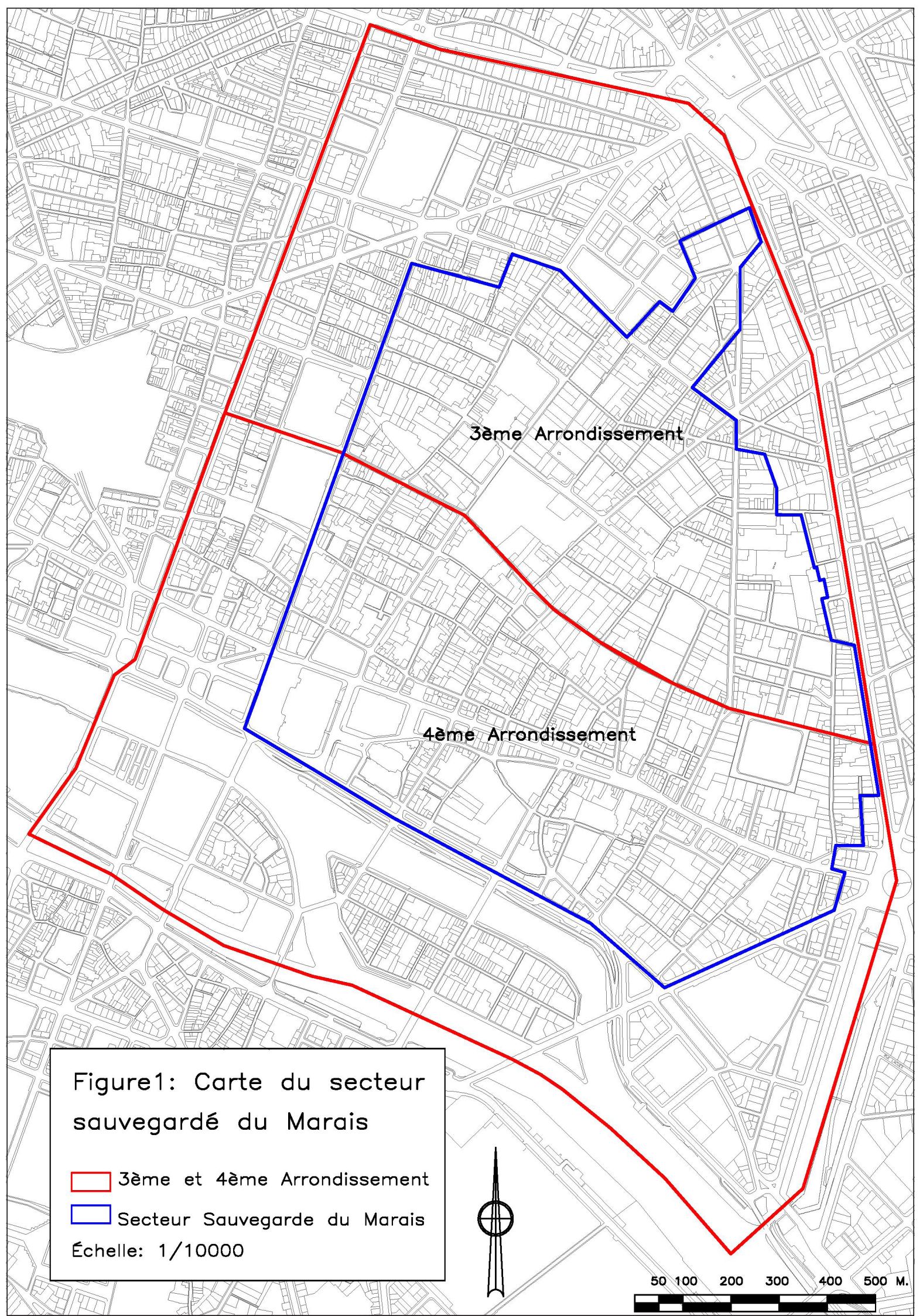


pérdida de impulso. Un lento declive comienza. "Sus iglesias y edificios eran transformadas en tiendas o destruidas. Los artesanos y los obreros de los barrios vecinos tomaban posesión de los grandes hoteles abandonados"16 (Bonnermeier, p. 14).

El emperador Napoleón $\mathrm{I}^{17}$ tenía algunas ideas para remodelar el centro de París. Así. hizo construir graneros de reserva en la parte sur-oeste del Marais y en los años 1830 el muelle de la Grève y del hotel de Ville fueron completamente reconstruidos y ensanchados. Luego, bajo el mandato de Napoleón III $^{18}$, el centro de París fue nuevamente remodelado gracias al Barón de Haussmann. ${ }^{19}$ Muchos antiguos edificios desaparecieron; sin embargo, una gran parte del Marais quedó intacta al ser un barrio pobre y sin interés.

En el siglo XIX, el Marais deviene en un barrio obrero, sucio, insalubre y en mal estado. Hasta antes de la Segunda Guerra Mundial (1939-1945) la situación sanitaria de la población fue deplorable. No solamente un gran número de hoteles fueron demolidos para construir en su lugar inmuebles con mayor cantidad de pisos, sino que empresas industriales y comerciales se instalaron en el barrio. Algunos años luego de la Segunda Guerra Mundial el Marais era el barrio más sucio y el de peor estado de París. Es recién en 1962 que la Ley Malraux salva al barrio. Al respecto, Andreas Bonnermeier indica que:

Para poder salvar el Marais, fue necesario suprimir la industria y el gran comercio, es decir 'rehacer todo el barrio para hacer una zona residencial. El Marais, de ese modo deviene en un barrio cada vez más buscado, acarreando al inicio numerosos talleres y depósitos 'estrechamente imbricados a los alojamientos', pero donde se desarrollaba un comercio especializado de cafés, restaurantes, boutiques de regalos, etc. Ciertos oficios tradicionales se mantenían, conservando en el Marais una real importancia económica para las actividades de joyería, óptica e instrumentos de óptica, 'artes de oficios', marroquinería y confección. (p. 19)

Finalmente, en 1990, el Marais se restaura con innombrables obras; además, muchos museos ocupan

16. Las traducciones de los textos-originalmente en francés- citados en el presente artículo han sido realizadas por el autor.

17. Napoleón I (1769-1821), Usó el nombre de Napoleón Bonaparte. Fue un general de la armada de la Revolución.

18. Napoleón III (1808-1873) fue el primer presidente de la República francesa, elegido el 10 de diciembre 1848.

19. Georges-Eugène Haussmann (1809-1891) fue prefecto del Sena de 1853 a 1870. Dirigió las transformaciones de París. los antiguos edificios del barrio. ${ }^{20}$ "Paulatinamente la gente busca un alojamiento en este barrio, ya que se encuentra 'a la moda' habitar el Marais. El Marais, así vuelve a devenir en un barrio chic y 'en boga', pero también caro (en cuanto a los alquileres)" (Bonnermeier. p. 22).

\section{De la protección de monumentos a la salva- guardia de los barrios históricos}

Chantal Ausseur Dolléans (2000) explica que:

Para numerosos teóricos y practicantes de la época, el patrimonio construido se limitaba a los monumentos, cuya protección era asegurada por la ley del 31 de diciembre de 1913 relativa a los monumentos históricos, extendida por la ley del 25 de febrero de 1943 a sus inmediaciones (p. 12).

La Ley del 31 de octubre de 1913 definía criterios de clasificación, de intervención obligatoria, etc. Esta apuntaba a la protección de monumentos históricos en ellos mismos y no a su entorno. Luego, la Ley de febrero del 1943 modifica la ley de 1913 y da paso a la noción de las abords de monuments historiques, inmediaciones de los monumentos históricos. Este impone una forma de vigilancia respecto de los proyectos de trabajo con un campo de visibilidad de 500 metros alrededor de un monumento histórico y en donde el Arquitecto de Edificios de Francia $(\mathrm{ABF})^{21}$ debe de deliberar su conformidad.

Luego de la Segunda Guerra Mundial, a finales de los años 50, una gran parte de los barrios y de los centros históricos de París estuvieron en estado de degradación e insalubridad. Ausseur (2000) encuentra que:

Era muy tentador aplicar a estos frágiles centros urbanos soluciones radicales preconizadoras por los partidarios de la ideología higienista: demoler y reconstruir según los principios de zonificación probados entonces sobre los barrios periféricos. La ciudad existente, con sus barrios por naturaleza confinados donde los vacíos y llenos son estrechamente imbricados, parecería ser ciertamente difícil de responder, a estos valores exclusivos de salubridad, de espacio y de circulación (p.14).

20. L'hôtel Aubert de Fontenay (le musée Picasso), I'hôtel de Lamoignon (la bibliothèque historique de Paris), I'hôtel Guénégaud (le musée de la Chasse et de la Nature), I'hôtel de Soubise (le musée de l'histoire de France et l'Archive national), I'hôtel Carnavalet (donde vive de 1677 à 1696 la Marquesa de Sévigné;existe un museo con los recuerdos de la Marquesa, muebles y testimonios de diferentes épocas).

21. Architecte de bâtiments de France. 
Durante los años 60, se producen numerosos acondicionamientos de los centros urbanos. André Malraux $^{22}$ toma conciencia de lo que la vitalidad urbana significa para la integralidad de los barrios históricos de la ciudad a partir de la experiencia del perímetro de restauración inmobiliaria de Tours en 1961/1962. Se promulga, entonces, la ley Malraux o Ley 62-903 el 4 de agosto de 1962, la cual instituyó la posibilidad de crear y delimitar los secteurs sauvegardés por parte del Estado. Malraux realiza un discurso y propone "salvar el alma de las ciudades y de cambiar la cara de Francia”. Explica la noción de conjunto urbano; la originalidad de su ley reside en "la creación de una herramienta de acondicionamiento que permita introducir en los inmuebles antiguos el confort moderno" y su objetivo principal es la protección y la puesta en valor del conjunto urbano que presenta un patrimonio mayor y particularmente degradado. Es el nacimiento de la protección de todo un sector urbano con una toma de consideración ligada a la armonía, unidad, coherencia y organización del conjunto urbano.

En seguida de la promulgación de Ley Malraux y del impacto cultural y artístico que representaba el festival del Marais ${ }^{23}$, en 1964, se creó el sector de salvaguardia del Marais, primer sector estudiado ${ }^{24}$ luego de la adopción de la ley Malraux; sin embargo, no fue aprobado hasta 1996, luego de 32 años de estudio.

En el expediente ${ }^{25}$ de la Dirección Regional de Asuntos Culturales (DRAC) ${ }^{26}$ de la Isla de Francia (2013), elaborado por el taller Blanc-Duché, se explica que:

Esta ley fue conocida para impedir que se siga la destrucción sistemática de los centros urbanos, organizado a nom-

22. George André Malraux (1901-1976), político, escritor e intelectual francés nombrado el 8 de enero de 1959, ministro de Estado encargado de los asuntos culturales.

23. El festival del Marais es un festival anual realizado entre 1961 y 1993. Ha sido creado a iniciativa de un grupo de ciudadanos reunidos alrededor de Michel Raude, a la misma fecha que la asociación para la salvaguardia y la puesta en valor del París histórico.

24. "Les premières études de secteurs sauvegardés ont été confiées à des Architectes en chef des monuments historiques, les seuls à cette époque, travaillant sur le patrimoine, mais avec une vision monumentale bien éloignée des problèmes de la ville dégradée et insalubre". Atelier Blanc-Duché, PSMV de Paris le Marais - Rapport de présentation préambule, 2013, p.4.

25. Dossier d'enquête publique sur le Projet de révision du Plan de Sauvegarde et de Mise en Valeur du Marais, dossier n³- partie III, justification des règles et la compatibilité avec les autres documents d'urbanisme.

26. Direction Régionale des Affaires Culturels bre de la renovación en el inmediato de la posguerra. Desde una veintena de años, la noción de patrimonio mismo evolucionó: Hoy en día se trata de volverlo una función viva, y no simplemente museal, intergrándolo en el urbanismo contemporáneo (p. 25).

\section{Marco de investigación}

Presentaremos, cronológicamente, la evolución del PSMV del Marais a través de los proyectos de los años 1969, 1976, 1980, 1996 y 2013 y de los procesos de revisión de1977, 1979-1980, 1984-1996 y 20062013. Ver el Gráfico 1 - Línea del tiempo comparativa (1960-2013). Para comodidad del lector, indicaremos los planes sucesivos bajo la forma PSMV según el año de su elaboración.

En 1962, el Marais deviene, por un lado, en el principal taller de París, el gran centro de "la fábrica y del negocio" y, por otro lado, en uno de los barrios el más vetusto e insalubre; era de algún modo el barrio menos ventilado y el más atestado de la capital francesa. $^{27}$

El primer proyecto, el Plan Permanente de Salvaguardia y de Puesta en Valor ${ }^{28}$ de Francia, sometido al Consejo de París en 1969 consideraba a la ciudad como un monumento y proponía una imagen ideal con relación a los habitantes y a las actividades. En estas condiciones, la idea de una modernización del Marais precedió a una idea de restauración de un barrio antiguo. El plan tenía una doble preocupación: trazar los ejes de penetración y alargar las vías de circulación y renovar las manzanas (insalubres y deterioradas). Este apuntaba a la conservación y preservación del "aspecto arquitectural tradicional del Marais, protegiendo las construcciones importantes, favoreciendo la restauración inmobiliaria y asegurando la homogeneidad de su entorno, y creando

27. Los espacios verdes representan $1.71 \%$ de la superficie. Los espacios libres son raros, pues la ocupación del suelo sobrepasa el 85 \% (55 \% para el conjunto de París). Las construcciones están "amontonadas" donde el aire y la iluminación penetran de manera parsimoniosa. Algunas manzanas alcanzan hasta 2000 habitantes por hectárea. El confort de los alojamientos es el peor de todos los distritos parisinos. El 30 \% está desprovisto de agua de grifo, el $60 \%$ sin WC y el $10 \%$ sin alumbrado eléctrico. Asimismo, elequipamiento social es insuficiente; hay $3 \mathrm{~m}^{2}$ de locales escolares para niños. (Paris Projet, p.47)

28. Utilizaremos también la denominación Plan Permanente de Salvaguardia y de Puesta en Valor (PPSMV) para designar el mismo procedimientos y querer fijar su permanencia. El nombre de la puesta en revisión del PSMV del Marais está para testimoniar que esta permanencia es relativa. 


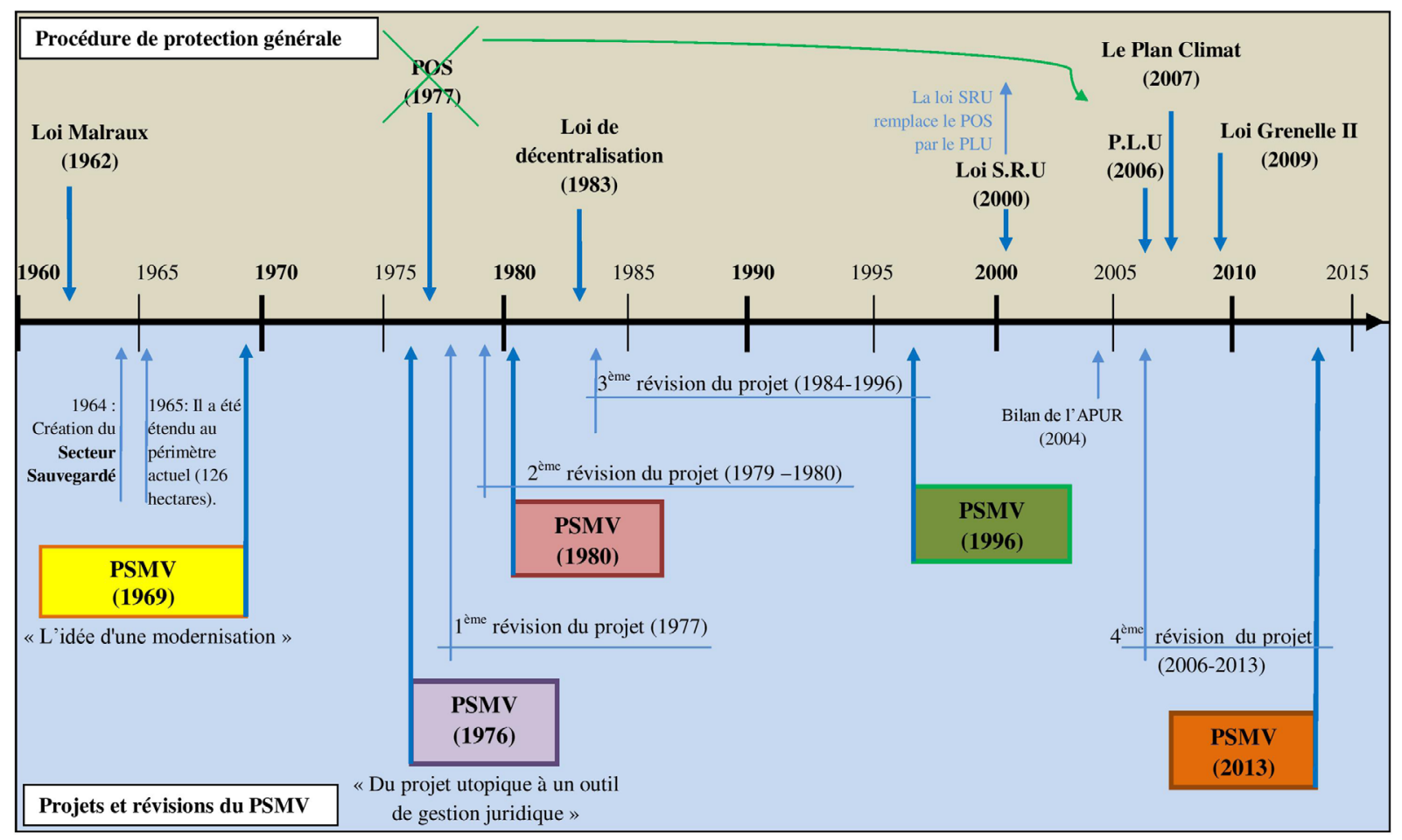

Gráfico 1.Línea de tiempo compartiva.

Elaboración. Shimabukuro. 2013.

condiciones más satisfactorias para la vida del barrio sus habitantes" (Paris Projet, 1970, p. 55).

Entre 1962 y 1971, un periodo intervencionista, se producen Proyectos de ciudad ideal, que se imponen a las ciudades y a los propietarios deseosos de intervenir sobre su patrimonio. Esta es la época de los îlots opérationnels, manzanas operacionales ${ }^{29}$ generados por una sociedad económica mixta cargada de operaciones, en la que el balance normalmente deficitario era encargado por el Estado (Atelier Blanc-Duché, 2013). Había sido estudiado "por manzana" y "por inmueble" y proponía una de las primeras imágenes globales que definía a largo término el devenir de un barrio histórico y se constituía en una guía para las modificaciones arquitecturales de la zona. Luego, a partir de 1968, comienza un periodo de atascamiento ${ }^{30}$ que se extiende hasta 1976.

29. De los 60 sectores salvaguardados puestos al estudio, 30 fueron objeto de "manzanas operacionales", en las cuales el principio de la renovación urbana (demolición/reconstrucción) fue frecuentemente aplicado. Atelier Blanc-Duché, PSMV de Paris le Marais Rapport de présentation préambule, 2013, p. 4.

30. " Plusieurs facteurs sont à l'origine de ce phénomène. En premier lieu, une antinomie entre une loi qui prône la conservation et la mise en valeur dupatrimoine bâti et des plans programmant
El PSMV de 1976 pasa “de un proyecto utópico a una herramienta de gestión jurídica”. Esta es una nueva versión del PSMV más razonable para hacer frente a las dificultades de mantenimiento de la población, las actividades ${ }^{31}$ y el realojamiento. Se propone: reducir los curetages $^{32}$ y las operaciones de renovación urbana, suprimir los ensanches de las vías todavía en vigor, reemplazadas en ciertos casos por los pasajes sobre arcadas en los edificios existentes. Este plan conservaba una parte muy importante de curetages, alrededor de 1000 propietarios sobre 1900 existen-

la destruction dîlots complets ou la création de vraisfaux espaces publics historiques, comme à Troyes où l'on voulait gommer la trame viairemédiévale en créant des galeries couvertes sous les immeubles à pan de bois que l'on auraitévidé sur un niveau. Mais le facteur le plus crucial est celui du manque de moyens, avec ladisparition de la politique des îlots opérationnels et des financements spécifiques". Atelier Blanc-Duché, PSMV de Paris le Marais-Rapport de présentation préambule, 2013, p.4 - p.5.

31. Luego de 1973 y del primer choque petrolero, el artesano entra en crisis. Empieza la desindustrialización textil; los oficios del metal y del mueble se encuentran en dificultad. Empieza así la gran mutación del Marais y será desde entonces ineludible.

32. Curetage es una palabra en francés utilizada para las operaciones de rehabilitación caracterizadas por la destrucción de las construcciones establecidas en los espacios interiores. (Saffache, 2005, p. 71) 
tes. Estas intenciones de curetages han tenido un rol importante en la desaparición de las actividades existentes, puesto que la rehabilitación o la transformación de los locales fueron, en numerosos casos, prohibidas.

En 1977, se realiza la primera revisión del proyecto. El grupo de trabajo se pronuncia para una mejor toma en cuenta de intenciones de acondicionamiento y una flexibilidad de ciertas reglas. Un diagnóstico general del proyecto es realizado para poner en evidencia los sectores de acondicionamiento del conjunto, los espacios verdes, calzadas para peatones y adaptación de reglas que fueron calcadas del Plan de Ocupación de los Suelos (POS) ${ }^{33}$ de París en particular la del sector salvaguardado ${ }^{34}$. (Atelier Blanc-Duché, 2013).

Luego, entre 1979 y 1980, se produjo la segunda versión del proyecto. En 1978, la ciudad de París asegura un mejor mantenimiento de las actividades artesanales e industriales que no se preveían en el plan. El concejo municipal adopta una orientación mayor para el plan de salvaguardia, que apuntaba a permitir el mantenimiento de las empresas industriales y artesanales en el barrio, mientras que el plan propuesto sugería la demolición de numerosos locales de actividades, y excluía su mejoramiento y su adaptación (Atelier Blanc-Duché, 2013). Fue deseable por el grupo de trabajo y por los arquitectos del Marais que el plan destaque mejor las intenciones de la colectividad (Kehren, Mahammodally et Schneider). El plan se centró en adaptar mejor las reglas arquitecturales de las especificidades del tejido del Marais, mejorar las intenciones de urbanismo, salir del raciocinio de la parcela $y$, por último, matizar los curetages estableciendo un dispositivo modulado destinado a evitar la desaparición sistemática de las actividades industriales y artesanales. Los objetivos de la revisión de los curetages fueron la separación de las inmediaciones de los monumentos históricos y de los conjuntos urbanos mayores y la creación de jardines en el centro de las manzanas, que fueron abandonadas o diferidas, con el fin de permitir el mantenimiento de actividades existentes o la rehabilitación de alojamiento.

\footnotetext{
33. Plan d'Occupation des Sols.
}

34. Notese, por ejemplo, el gálibo de las construcciones futuras y la posibilidad de transformación con motivo de rehabilitación. Atelier Blanc-Duché, PSMV de Paris le Marais - Rapport de présentation préambule, 2013, p.13.
El PMSV de 1980 presentó "modificaciones en lo concerniente a los curetages, reglas de la arquitectura e inserciones urbanísticas" (Kehren, Mahammodallu et Schneider, (s.f), p.23), se produjo la aprobación del documento de reexaminación completa del plan. ${ }^{35}$ Se realizan nuevas elaboraciones en razón de la evolución del tejido urbano y de la extinción del sector. Dos tipos de operaciones modificaron la estructura urbana del Marais . Por una parte, la apertura de nuevas vías y las definiciones de alineaciones y por otro lado, las operaciones aisladas. Entre 1984 y 1996, luego de varios años de estudio y de análisis del barrio, se encomendó al arquitecto Bernard Wagon, en 1984, la elaboración de la revisión del PSMV. Este nuevo PSMV fue publicado en 1992, puesto en encuesta pública en 1994 y aprobado por el decreto del 23 de agosto de 1996. Se considera que esta fue la tercera revisión del proyecto.

El PSMV de 1996 es el resultado de un ajuste de la salvaguardia del patrimonio, presente desde la ley Malraux y los primeros estudios del PSMV y la información expresada en los reportes de encuesta pública de 1994. "La atención de los residentes del Marais para el valor histórico, arquitectural y urbano del barrio y el deseo de la Ciudad de París de mantener el carácter evolutivo de sus lugares de actividades". En lo que concierne a las nuevas disposiciones, el proyecto propone la introducción del "subsector de acondicionamiento de conjunto" en el plan. La particularidad es reportar sobre el plan en relación al perímetro de acondicionamiento dotado de un margen reglamentario más largo en materia de construcción nueva y la separación de dos situaciones particulares específicas. Por una parte, la protección, la puesta en valor y la restauración del patrimonio arquitectural de los edificios existentes ${ }^{36}$ y por otra, las posibilidades de construcciones nuevas, extensiones y modificaciones relativas a la volumetría y a la implantación, lo que presentaba una mejor apertura hacia la arquitectura contemporánea. Así, el Atelier Blanc-Duché en 1996 explica que los ajustes son traducidos en:

La actualización de los documentos gráficos, en particular los relacionados a las operaciones de curetage o construcción. Los curetages mantenidos son de los

35. Realizado por un grupo de trabajo compuesto por arquitectos del PSMV, asociados al I'Atelier parisien d'urbanisme, la Direction de l'aménagement urbain de la ville de Paris y los Architectes de Bâtiments de France.

36. Permite todas las iniciativas que apuntan a restituir las disposiciones históricamente conformes para las edificaciones antiguas. 
inmuebles cuya demolición podrá ser impuesta con motivo de operaciones de acondicionamiento público o privado.

La determinación de "fachadas a conservar" fue extendida a ciertos ejes principales: ${ }^{37}$ la conservación en razón del interés arquitectural, pintoresco o de su carácter de edificio de acompañamiento.

El reexamen de ciertos artículos de reglamento permitió una mejora de la presentación de la redacción del documento y un ajuste de ciertos disposiciones a la luz de la experiencia adquirida.

El mejoramiento de la redacción del reglamento en cuanto a las generalidades, a las definiciones y a la instrucción de los archivos.

- El mejoramiento de la definición de los espacios libres y las reglas que los afectan. La introducción, en documentos gráficos, de los espacios protegidos a título de "patios pavimentados" con el fin de tener una armonía urbana del centro de la manzana.

El conjunto de dispositivos de protección y de puesta en valor es mantenido; sin embargo, una flexibilidad de las reglas de construcción fue introducida para satisfacer la evolución del habitante, el confort y la seguridad.

- La introducción al plan de prescripción que apuntaba a proteger las perspectivas urbanas del siglo XIX. ${ }^{38} \mathrm{La}$ mayor parte de los inmuebles interesantes datan de la época comprendida entre 1850 y 1930.

Luego del PSMV (1996), han aparecido ciertas leyes, particularmente la ley relativa a la Solidaridad y a la Renovación Urbana (SRU) ${ }^{39}$ del 13 de diciembre del 2000, que prevé la desaparición del Plan de Ocupación del Suelo (POS) ${ }^{4041}$ de 1997 e instituye el Plan Local de Urbanismo (PLU) ${ }^{42}$ de junio del 2006.

37. La calle Rivoli, la calle François Miron, la calle de los Archives (en parte) y, en la periferia del sector, el boulevard Henri IV (costado de números impares), cuyos inmuebles presentan una riqueza arquitectural excepcional.

38. "La protection des perspectives constituées par les immeubles du XIXème siècle a été notablement étendue, dans l'objectif de maintenir la cohérence des façades. La protection de ces édifices est limitée aux façades uniquement, et vise à interdire l'altération de l'unité assurée par l'échelle des immeubles et la modénature qui les relie les une aux autres." Atelier Blanc-Duché, PSMV de Paris le Marais - Rapport de présentation préambule, 1996, p.13.

39. Loi relative à la Solidarité et au Renouvellement Urbains.

40. Plan local d'urbanisme.

41. Un documento de urbanismo cuyo régimen ha sido creado por la ley de orientación territorial de 1967. El POS subsiste y guarda toda su validez jurídica, tanto que no se transformó en PLU.

42. Es un documento estratégico al mismo tiempo que una he-
El urbanismo se vuelve el centro de la reflexión y así los sectores salvaguardados se inscriben en una lógica urbana de conjunto más amplia. Esta reforma de derecho del urbanismo generó la necesidad de analizar la compatibilidad del PSMV con los otros documentos de urbanismo. Por otro lado, teniendo en cuenta que el contexto económico que había evolucionado desde los años 1960 , el Consejo de París, durante las sesiones del 24 y 25 de junio del 2002, pide al Estado la revisión del PSMV del Marais y del séptimo arrondissement (Blancot, 2004). A continuación, ese consejo, durante la sesión del 27 de enero del 2003, examinó el documento de orientación para la elaboración del Proyecto de Acondicionamiento y de Desarrollo Sostenible (PADD) ${ }^{43}$, incluido en el PLU, el cual indica que: "Los dos territorios cubiertos por los planes de Salvaguardia y de puesta en valor del Marais y del séptimo arrondissement son concernietnes para las nuevas orientaciones definidas en el PADD. Más allá de la necesaria preservación de un patrimonio excepcional, las disposiciones deben ser tomadas para reforzar la dinámica de los barrios, permitir a sus habitantes mantener y frenar así los fenómenos de museificación" (Blancot, 2004, p.1). Así,de octubre del 2003 a febrero del 2004 se realizó un Balance sobre las dificultades de gestión y de aplicación. Mejoramiento y modernización necesarias por el Taller Parisino de Urbanismo (APUR) ${ }^{44}$. El estudio resalta que por una parte es urgente completar el PSMV para armonizar el PADD y el futuro PLU de París, y por otra parte preconiza que sean lanzados, al mismo tiempo, estudios urbanos y patrimoniales indispensables para actualizar el PSMV y pronunciarse sobre su extensión.

Luego, la revisión del PSMV fue encargada por orden ministerial del 15 de junio del 2006, por la Comisión Local del Sector Salvaguardado (CLSS) ${ }^{45}$, creada por orden prefectoral del 19 de febrero del 2009 y compuesta por el alcalde de París y el prefecto del Consejo de París, representantes del servicio del Estado, y

rramienta reglamentaria. Comprende un reporte de presentación del contexto y de las perspectivas de evolución de la ciudad, un Proyecto de Acondicionamiento y de Desarrollo Sostenible (PADD) que define las orientaciones de urbanismo y de acondicionamiento para todo el territorio Este fija las reglas de utilización de los suelos ilustrados para los documentos gráficos (mapas, planos, esquemas) y los documentos técnicos anexos. Mairie de Paris, Direction de l'Urbanisme, Op.cit, p.4.

43. Projet d'Aménagement et de Développement Durable

44. Atelier Parisien d'Urbanisme.

45. Commission Locale du Secteur Sauvegardé 
por personalidades calificadas. Esta comisión validó los trabajos de los estudios, el proyecto de PSMV y propuso el "estudio de arquitectura-urbaniso taller Blanc et Duché asociando las competencias en temas de historia, património, economía, [...] para construir los estudios necesarios a la revisión del PSMV [...]" (Mairie de Paris, 2013).

En mayo del 2009, un documento provisional - una fase del estudio que muestra un primer avance del documento final- de la revisión del PSMV del Marais es presentado por el taller Blanc et Duché. Los grupos de trabajo tuvieron por objetivo estimular la reflexión, mostrando un máximo de información, con la participación de expertos sobre diferentes temas tales como: la armonía PLU-PSMV, el espacio público, los patios interiores y el desarrollo sostenible.

Después, entre el 2009 y el 2010 se realiza una "concertación en el tercer y cuarto arrondissements" a lo largo de la elaboración del documento. Así, hubo reuniones públicas, talleres temáticos, conferencias, etc. para tomar conocimiento de las expectativas de cada una y reunir los diferentes puntos de vista. El resultado del trabajo participativo es un Balance de las reuniones públicas de concertación previa legal realizado el 05 de noviembre del 2012, el cual tiene por objetivo forjar proposiciones para el PSMV en tres sectores diferentes: patrimonio, que se refiere a la preocupación para proteger numerosas construcciones del siglo XIX y XX y el número de las prescripciones de demolición las cuales disminuyeron: el desarrollo sostenible que con la prolongación de la ley Grenelle II produjo un cambio sobre la superficie mínima y las reglas de altura de construcción para la instalación de dispositivos de energía renovable $\mathrm{y}$, finalmente, las funciones urbanas (alojamiento, equipamiento, comercio), la diversidad social y la protección y fomento del comercio y artesanía (rol de actividades y vida del barrio).

Del 18 de febrero al 22 de marzo del 2013, se realizó una encuesta pública en las municipalidades del tercer y cuarto arrondissements, la cual tomó en cuenta los elementos resultantes de la concertación aprobada por la CLSS, previo dictamen de la Comisión Nacional de los Sectores Salvaguardados (CNSS) ${ }^{46}$. Durante la encuesta, el público tomó conocimiento del documento y anotó sus observaciones, propuestas y contra-propuestas sobre el registro.

46. Commission Nationale des Secteurs Sauvegardés
Finalmente, el PSMV (2013) fue aprobado por orden del prefecto de la región de la Isla de Francia -prefecto de París - el 18 de diciembre del 2013. Este responde a un mejor conocimiento de la protección del patrimonio. Se adapta mejor a las tipologías históricas de los edificios protegidos, con el fin de poner en valor y contribuir al: equilibrio del hábitat/empleo y diversidad social, a la calidad de vida de los barrios y a la preservación del medio ambiente de las orientaciones del PADD.

\section{Estado de la cuestión: desarrollo de la investi- gación}

Hemos encontrado un buen material de trabajo, muy preciso. Las informaciones concernientes al Marais son ricas y se intensifican día tras día. Con relación a la información del PPSMV de Francia, tomamos como punto de partida los archivos privados de la Médiathèque de l'Architecture et de l'Patrimoine en la que se encuentran todos los archivos de los estudios operacionales para el PPSMV de 1968. Consultamos también la Bibliothèque du Pavillon de l'Arsenal - de donde extrajimos particularmente el contenido de la revista bimestral PARIS PROJET. Aménagement Urbanisme Avenir es un artículo preparado a partir del reporte establecido por el equipo de arquitectos Louis Arretche ${ }^{47}$, Michel Marot ${ }^{48}$ y Bernard Vitry asistentes de Maurice Minost, encargados de la elaboración del Plan del Marais y de la Memoria presentada por el prefecto de París a la Asamblea Municipal en octubre de 1969.

También consultamos la Biblioteca Nacional de Francia, la Bibliothèque Cité de l'Architecture et du Patrimoine (Palais de Chaillot), el Centre de Ressources Documentaires Aménagement, Logement, Nature (CRDALN) -ex Centre de Documentation de l'Urbanisme (CDU)-, Association pour la Sauvegarde et la Mise en Valeur du Paris historique (ASMVPH), la École Nationale Supérieur d'Architecture Paris Val de Seine (ENSAPVS), Université Paris Diderot - Paris 7 y Bibliothèque Historique de la Ville de Paris (BHVP).

Finalmente, la investigación al respecto fue sustentada el 18 de junio del 2013; por lo tanto, como el PSMV (2013) fue aprobado el 18 de diciembre del

47. Louis Gerald Arretche (1905-1991) es un arquitecto y urbanista francés.

48. Michel Marot (1926) es un arquitecto francés. 
2013, nuestra investigación alcanza hasta la encuesta pública realizada en las municipalidades del tercer y cuarto arrondissements de París, del 18 de febrero al 22 de marzo del 2013. Así, hemos utilizado el PSMV que estuvo todavía en revisión y que no ha tomado en consideración el reporte del commisaire enquéteur.

\section{Metodología}

El análisis del PSMV muestra un constante desfase entre los objetivos definidos por el marco jurídico y las aplicaciones.

En este sentido, en el marco del estudio de la política de protección y de valorización del patrimonio, hemos analizado todos los aspectos que conciernen a la evolución del PSMV con el fin de profundizar y extender el alcance en un nuevo documento y tratar de encontrar una respuesta a la preocupación que resulta de esta evolución. Por esta razón, y a partir de este procedimiento de evolución, nos hacemos la pregunta. ¿Cómo estos objetivos y reglamentos han ido evolucionando? Constatamos que en el transcurso de estos cuarenta últimos años la zona cubierta por el PSMV ha experimentado profundas evoluciones.

Hoy en día, es impensable realizar un PSMV sin tener en cuenta los efectos sobre la dinámica urbana del territorio en cuestión. Por lo tanto, este análisis tiene por objetivo hacer evidente lo siguiente: la evolución histórica del barrio del Marais. La evolución del procedimiento de protección general, es decir, la evolución del procedimiento de protección del patrimonio a nivel de los documentos jurídicos de la ciudad de París.

La evolución del procedimiento de protección específico; es decir, la evolución del procedimiento de los proyectos del PSMV para mostrar la problemática y la proposición de cada proyecto del PSMV y los numerosos y constantes estudios de revisión del PSMC desde el inicio hasta nuestros días, mostrando los testimonios de la evolución de la teoría a la práctica.

El análisis crítico y comparativo de los documentos gráficos y documentos textuales del PSMV. Un método de análisis gráfico realizado a partir de la creación de una serie de cuadros sinópticos con el fin de facilitar la comprensión del tema lo que proporciona una estructura global y coherente de las numerosas relaciones del tema.

\section{Resultado}

El PSMV ha evolucionado considerablemente. Con el fin de hacer un análisis claro y conciso, hemos encontrado oportuno hacerlo en tres partes. En primer lugar, analizamos los objetivos iniciales del primer proyecto del PSMV (1969). En segundo lugar, analizamos la evolución de los objetivos de los diferentes proyectos del PSMV (1969, 1976, 1980, 1996 y 2013) y en tercer lugar, analizamos los objetivos del PSMV (2013) haciendo una comparación más precisa con los documentos textuales (reglamentarios) y gráficos (cartografía) del PSMV (1996) (gráfico 2, diagrama de evolución de los objetivos del PSMV).

\section{Los objetivos iniciales del primer proyecto PSMV de 1969}

Con el objetivo de dar una solución a la problemática de salubridad y de higiene en el Marais, el PSMV planteó ciertos objetivos desde sus orígenes. Identificamos y enumeramos cinco los que se relacionan entre sí. Ver las casillas de color amarillo en el Gráfico 2 - Diagrama de evolución de los objetivos del PSMV.

Objetivo 1: conservación de la arquitectura tradicional y aseguramiento de la homogeneidad. Hay necesidad de saber identificar los edificios a proteger y los edificios a demoler.

- Objetivo 2: plan de curetage. Se refiere a una operación de rehabilitación urbana que tiene por objetivo obtener la restauración de una manzana urbana antigua, según la cual se destruye un inmueble salvo la fachada cuando este tiene un interés histórico o arquitectural y se reconstruye integralmente su parte trasera. Es una intervención en materia de higiene, confort y autenticidad estética.

- Objetivo 3: reanimación a través de funciones nuevas. Se refiere a compensar las funciones desaparecidas por la demolición a fin de dar una nueva actividad a los edificios conservados.

- Objetivo 4: crear condiciones más satisfactorias para la vida del barrio y sus habitantes.

- Objetivo 5: trazar ejes de penetración y ensanchar las vías de circulación.

Gráfico 2. Diagrama de evolución de los objetivos del PSMV.

Elaboración. Shimabukuro. 2013. 

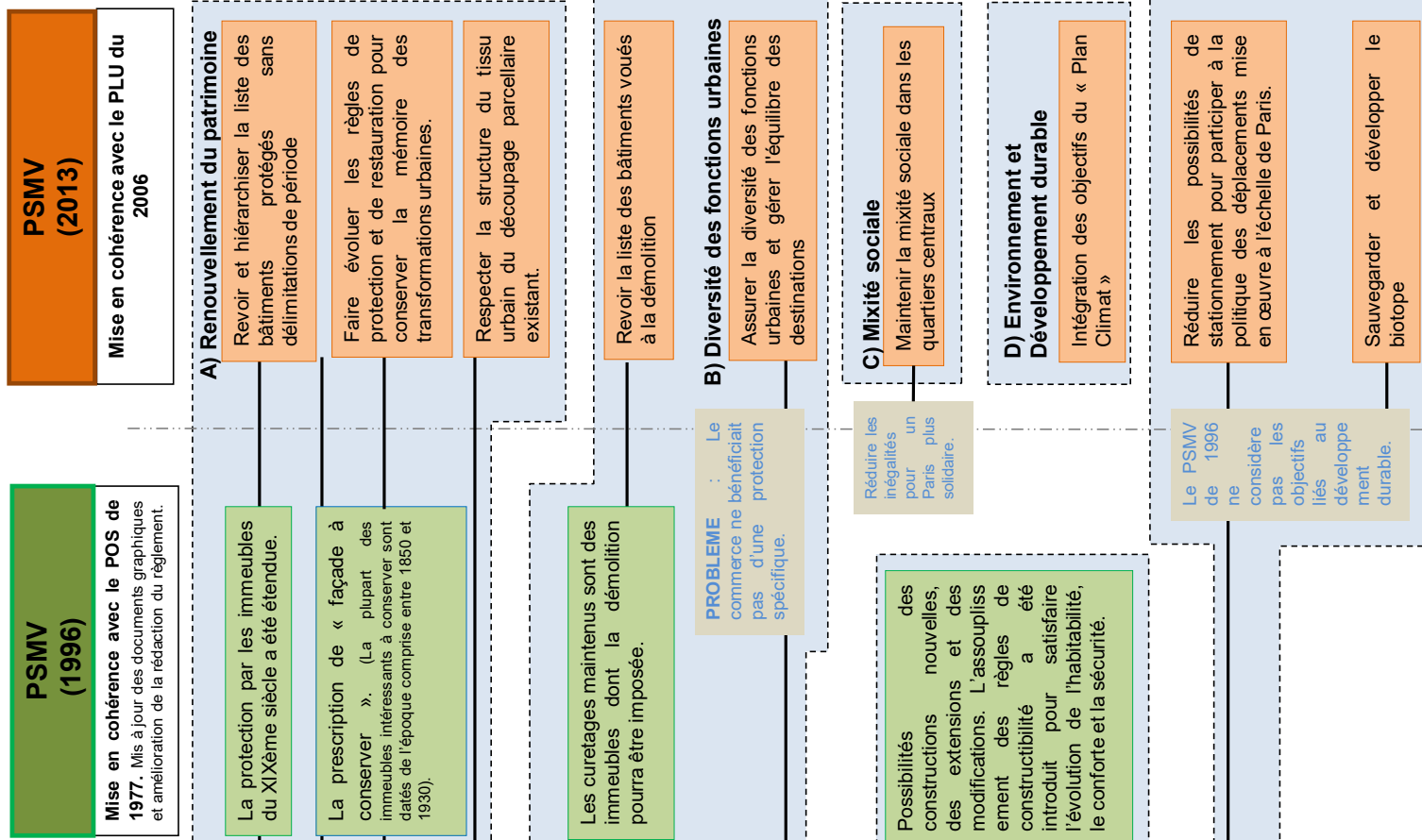

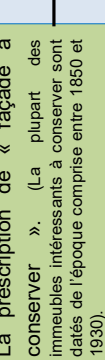
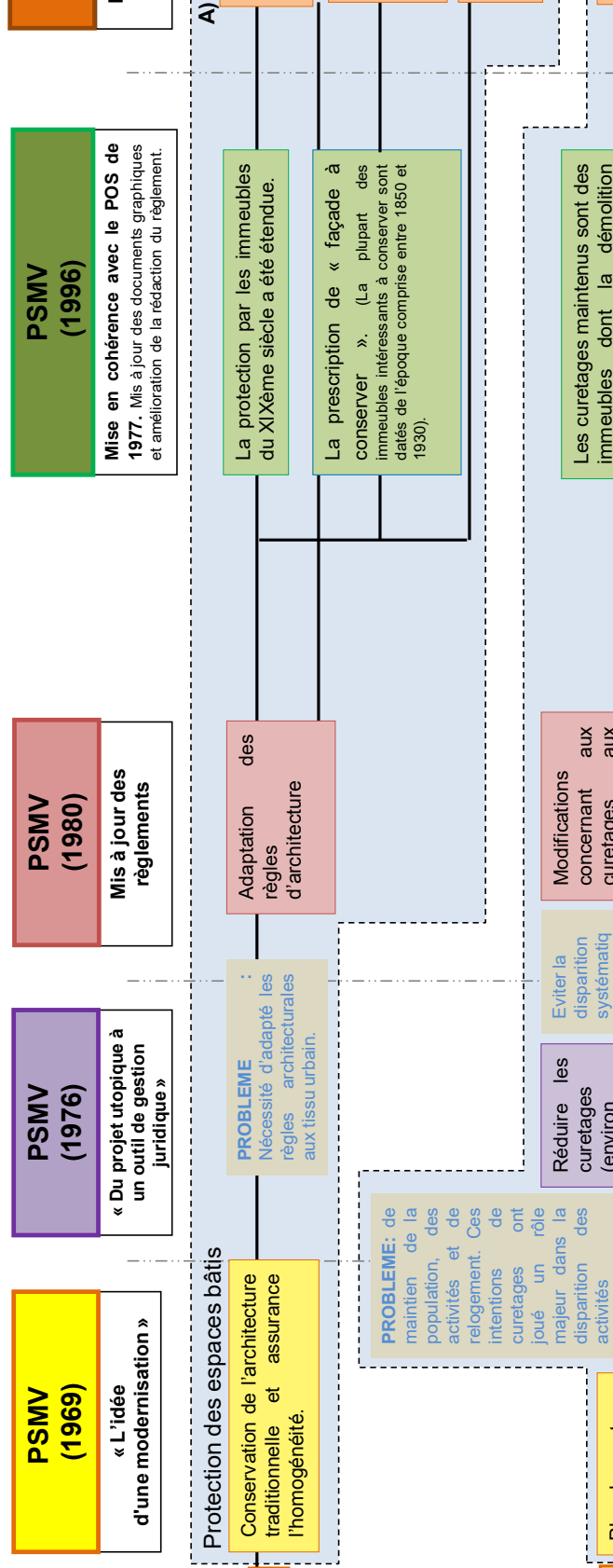


\section{La evolución de los objetivos de cinco proyectos del PSMV (1969, 1976, 1980, 1996 y 2013)}

Esto corresponde a la evolución de todos los proyectos del PSMV. Conforme analizamos, fuimos constatando que hubo tres tipos de objetivos: adaptados, detenidos (objetivos todavía existentes, pero que no han evolucionado) y agregados (objetivos de desaparecieron luego del primer proyecto).

\subsection{Los objetivos adaptados}

Vimos que de cinco objetivos propuestos en el primer proyecto, tres evolucionaron hasta el PSMV (2013)

A partir de esta perspectiva, presentaremos cada evolución según la enumeración de los objetivos a continuación.

Conservación de la arquitectura tradicional y aseguraramiento de la homogeneidad (objetivo 1). Observamos que la originalidad del plan fue la conservación de los aspectos arquitecturales tradicionales del Marais, protegiendo las construcciones importantes y asegurando la homogeneidad de su medio. La leyenda del primer documento gráfico, que concentra los edificios a conservar o a proteger, fueron categorizados de la siguiente manera: inmueble protegido a título de Monumento Histórico ( $\mathrm{MH})$, inmueble interesante a proteger, inmueble de acompañamiento a conservar y fachadas particularmente interesantes. Sin embargo, comienza a aparecer la necesidad de adaptar las reglas arquitecturales al tejido urbano, de manera que el PSMV (1980) actualiza la reglamentación y propone las adaptaciones de sus reglas de arquitectura. Luego, el PSMV (1996) extiende la protección a los inmuebles del siglo XIX y las fachadas pasan de ser "fachada particularmente interesante" a ser "fachadas a conservar". Por último, el PSMV (2013) tuvo los objetivos de jerarquizar la lista de los edificios protegidos con delimitación de periodo, hacer evolucionar las reglas de protección y de restauración para conservar la memoria de las transformaciones urbanas, y respetar la estructura del tejido urbano de recorte de parcelas existentes.

Plan de curetage (objetivo 2). Teniendo en cuenta el problema de la densidad de ocupación del suelo, el PSMV (1969) propone un plan de curetage y de despeje del centro de las manzanas. Fue un proyecto que proponía reconquistar progresivamente, en el corazón de las manzanas o en los patios de los inmuebles, los espacios libres indispensables para la ventilación del Marais. Por eso, el PSMV (1969) propuso los edificios cuya demolición era deseable o certera a fin de recu- perar el suelo para priorizar el acondicionamiento vegetal con jardins continus, jardines continuos, creados en el corazón de las manzanas y abiertos al público. No obstante, esta proposición fue utópica; de manera que el PSMV (1976) redujo los curetages a 1000 propietarios de los 1900 existentes. Como el PSMV (1980) a fin de evitar los problemas de desaparición sistémica de las actividades industriales y artesanales, el había planteado modificaciones concernientes a los curetages, en las reglas de arquitectura y en las intenciones de urbanismo, en 1996 el curetage cambio de ser "demolición deseable" y "demolición certera" a ser "inmueble cuya demolición podrá ser impuesta salvo en caso del mantenimiento de las actividades industriales, artesanales o comerciales". Finalmente, en el PSMV (2013) se propuso revisar la lista de los edificios condenados a la demolición.

Trazar los ejes de penetración y expandir las vías de circulación (objetivo 5). La circulación es un aspecto importante para el buen desarrollo de la ciudad. Al inicio, se impuso una política favorable al automóvil el cual fue seguido hasta el PSMV (1996). Sin embargo, hoy en día, es el PADD quien propone una política de desplazamiento. Desde esta perspectiva, el PSMV (2013) propone reducir las posibilidades de estacionamiento para participar en la política de desplazamiento aplicado a la escala de París.

\subsection{Los objetivos en los que la evolución se detuvo}

Se refiere a los objetivos existentes, pero que no han tenido la necesidad de ser revisados.

- Reanimación a través de las funciones nuevas (objetivo 3). El objetivo es provocar la reanimación y encontrarle funciones nuevas correspondientes a su capacidad interna y decoración.

Creación de condiciones más satisfactorias para la vida del barrio y las de sus habitantes (objetivo 4). Aparentemente todas las intervenciones fueron realizadas buscando crear condiciones más satisfactorias para la vida del barrio y de sus habitantes.

\subsection{Los objetivos agregados desde el PPSMV}

La aparición del POS (1977) produjo la armonización del PSMV con su documento. La creación de nuevos objetivos se dio a partir del PSMV (1996). Los nuevos objetivos fueron: la flexibilidad de las reglas de construcción -introducción para satisfacer la evolución de la habitabilidad, el confort y la seguridad - y las posibilidades de construcciones nuevas. Luego, la incorporación del PADD del PLU, en el 2011, produ- 
jo nuevos objetivos en el PSMV (2013) relacionados al desarrollo sostenible y a la mezcla social. Así, los nuevos objetivos son: mantener la mezcla social en los barrios centrales, la integración de los objetivos del Plan Climat, salvaguardar y desarrollar el biotopo. ${ }^{49}$

\section{Los objetivos del PSMV (2013)}

Ver las casillas en color naranja en el gráfico 2 - diagrama de evolución de los objetivos del PSMV.

\section{A) La renovación del patrimonio}

La lista de edficios fue extendida, debido a una noción de protección sin delimitación de periodo

Nueva clasificación de la utilización del suelo. La integración a la lista de esta diversidad de edificios ha producido dos tipos nuevos de categorías: tipo $\mathrm{A}^{50}$ y tipo $\mathrm{B},{ }^{51}$ caracterizadas por su interés patrimonial. Cada uno de los edificios fue objeto de un análisis (de descripción y de apreciación fundada sobre la documentación histórica, visitas, reportajes fotográficos y fichas de encuesta) que apuntaba a determinar su valor patrimonial.

Importante creciente, del $50 \%$ al $78 \%$, de los edificios protegidos. Esto es debido a la integración del patrimonio del siglo XIX y moderno. Según el inventario del Marais de 1970, 75 \% de los inmuebles que existían en el interior del perímetro del sector de salvaguardia fueron construidos antes de 1870 (Paris-Projet, 1970). El PSMV (1996) se concentró en el patrimonio monumental y en el del antiguo régimen, el del siglo XIX y XX, que particularmente había sido mal protegido (Christiane Blancot, 2004). Por consecuencia, el PSMV (2013) cubre un largo espectro histórico desde el siglo XVI hasta inicios de los años $1970^{52}$.

49. "Milieu biologique déterminé offrant à une biocénose des conditions d'habitat relativement stables". REY Alain, Le Petit Robert, 2011.

50. Tipo A: inmueble o parte del inmueble a conservar, cuya demolición eliminación o alteración son impedidas y cuya modificación es sometida a condiciones especiales.

51. Tipo B: inmueble o parte del inmueble a conservar, cuyo acondicionamiento puede comportar intervenciones sobre la estructura y/o sobre la repartición de los volúmenes interiores existentes. Es autorizada a partir de ciertas condiciones.

52. "Bâtiments d'habitation, hôtels, ateliers, bâtiments d'activités, immeubles de bureaux, marchés, entrepôts, locaux commerciaux, restaurants et cafés .... [...] Leur diversité reflète la reconnaissance de nouvelles catégories de patrimoine jusqu'ici insuffisam-
Por otro lado, en lo que respecta a los inmuebles o parte de los inmuebles de protección con la denominación de Monumentos Históricos, observamos un descenso de $8 \%$ a $3 \%$. Esta disminución es artificial, porque en el antiguo reglamento, las protecciones de un elemento particular (fachadas, escaleras, chimeneas, etc.) eran resultado de la incorporación automática de todos los edificios en el conteo de los terrenos protegidos. En este sentido, la proporción de los edificios no protegidos disminuyó de 36 \% $19 \%$.

La lista de los edificios condenados a la demolición disminuyó. La lista de 1996 fue realizada en función a la idea inicial de curetage, en coherencia con la obligación de luchar contra la insalubridad que había provocado la concentración de actividades industriales desde hace un siglo y la degradación de las condiciones del hábitat.El PSMV (2013) realizó una revisión, caso por caso, en función de los retos que se vinculan a cada parcela ${ }^{53}$ con el fin de tener más en cuenta las actividades y conservar los diferentes tipos de ocupación de las parcelas.

Cambio de la superficie. El número de edificios cuya demolición puede ser impuesta disminuyó en casi una 5 ta parte (de $14 \%$ a $3 \%$ ).

\section{B) Diversidad de las funciones urbanas}

Reforzamiento de la protección del comercio. En el PSMV (1996) el comercio no fue beneficiado por una protección específica. El PSMV (2013), a fin de imponer la construcción de locales comerciales o de evitar una desestructuración del tejido comercial, propone en el reglamento una serie de artículos:

Artículo US.2.2.a.2: los locales situados en la planta baja deben (en caso de construcción, reconstrucción o rehabilitación pesada) ser destinados al comercio o a la artesanía (a excepción de de los locales de acceso a los inmuebles)

ment prises en compte : I'habitat vernaculaire caractéristique des siècles passés, l'architecture industrielle, les opérations de lotissement spéculatives du XIXème siècle, les grands courants esthétiques du XXème siècle jusqu'à la période des Trente Glorieuses". Atelier Blanc-Duché, PSMV de Paris le Marais - Dossier N 3. Partie III Justification des règles et la compatibilité avec les autres documents d'urbanisme. Op.cit, 2013, p. 28.

53. Los locales de actividades a proteger, patrimonio a reconocer y proteger, tipologías de ocupación de los fondos de parcelas a alentar, evolución de los usos de higiene y seguridad de las construcciones vecinas. 
- Artículo US.2.2.a.3: Se prohibe el cambio de destinación de los locales comerciales o artesanales en planta baja. (Atelier Blanc-Duché, 2013).

\section{C) Mezcla social}

Aumento del porcentaje de la superficie destinada a la mezcla social: para mantenerla en los barrios centrales, el artículo US.2.3 del reglamento de PSMV (2013) fija una norma que determina un $30 \%$ de alojamiento social, superior al objetivo de $25 \%$ fijado por la ley SRU. Este porcentaje superior marca la voluntad de actualizarse con relación a la situación de déficit actual.

\section{D) Medio ambiente y desarrollo sostenible}

Integración de nuevos dispositivos que autorizan el establecimiento de dispositivos técnicos: hemos constatado que la ciudad de París ha adaptado el Plan Climat el 1ro de octubre de 2007 con el objetivo de reducir el conjunto de emisiones de gas de efecto invernadero de su territorio (Atelier Blanc-Duché, 2013, p.29.). De esta manera, el PLU integró las nuevas disposiciones que autorizan la aplicación de los dispositivos técnicos en las construcciones nuevas o existentes, a fin de economizar o de producir energía renovable. "Estas acciones se refieren por ejemplo a la renovación de los edificios, las energías renovables, desplazamiento, gestión de desechos." (Mairie de Paris, 2009, p. 4)

Reducción de las posibilidades de estacionamiento para participar en la política de los desplazamientos ejecutados a la escala de París: el taller Blanc-Duché explica, en el 2013, que para compatibilizar el desfase en cuanto a las normas entre el PSMV (1996), que tiene una política favorable al automóvil, ${ }^{54}$ y la nueva política de desplazamiento del PADD:

Hacer respirar París: una nueva política de desplazamiento que tiene como objetivo la limitación del estacionamiento, el PSMV revisado ${ }^{55}$ propone una política de disuasión explicando que "la política de protección del patrimonio deberá beneficiar más a sus nuevas disposiciones porque la obligación de creación de lugares de estacionamiento fue una obligación fuerte en el tejido urbano denso, constitui-

54. Hay que recordar que este plan radica en la necesidad de imponer el acondicionamiento de un número mínimo de lugares de estacionamiento para solucionar la demanda de estacionamiento en la vía pública.

55. Cette source est un document préalable à l'approbation du PSMV (2013). Si bien que, le PSMV révisé fait référence au PSMV (2013). do por numerosas parcelas estrechamente comunicadas, por vías poco anchas, con un sub-suelo frecuentemente muy ricas sobre el plan arqueológico. Así el PSMV revisado impide toda creación de acceso para parques de estacionamiento al borde de vías de menos de 8 metros de ancho (p. 11.)

Queremos agregar otros aspectos (tratados en la página 34 y 35 del mismo documento) relacionados a esta política de disuasión:

La facilitación del uso de los transportes colectivos y la reducción de la utilización del automóvil en la ciudad. En este aspecto, el PSMV (2013) propone tratar de manera diferente el estacionamiento vinculado al domicilio y los estacionamientos ligados al empleo.

"La posibilidad de un estacionamiento cerca del domicilio debe ser ofrecido para permitir que los residentes no estén obligados a efectuar un desplazamiento del domicilio al trabajo en automóvil. En efecto, igualmente es necesario no alentar la posibilidad de ofrecer un estacionamiento cerca del lugar de trabajo, en particular en los sectores muy bien comunicados por el transporte colectivo" (Atelier Blanc-Duché, 2013).

- La exigencia de un mínimo de 2,25\% de la superficie de suelo local (vivienda y oficina) para el estacionamiento de bicicletas y cochecitos. Esta disposición favorece el desarrollo de una oferta privada de estacionamiento.

Adaptación de reglas para salvaguardar y desarrollar el déficit de biotopo: el PADD tiene como fin preservar el paisaje urbano, mejorar la calidad y el marco de vida de los habitantes, salvaguardar y desarrollar el biotopo. Por eso, los dispositivos del PLU que apuntan a crear espacios libres suplementarios y patios internos deben ser aplicados. Sin embargo, constatamos que la mayor parte de los espacios no edificados del Marais están constituidos por patios pavimentados; es decir, que solamente los hoteles particulares importantes poseen un jardín en la parte trasera del cuerpo principal de la vivienda. Hay un 9.9 hectárea de patio (equivalente al $55 \%$ ) y 8.3 hectárea. de jardín (equivalente al $45 \%$ ). Los cálculos muestran que el Marais constituye un sector de déficit de biotopo. Existe solamente el $8 \%$ de espacio libre dedicado al aspecto vegetal en relación a la superficie total de las parcelas. Así, para evitar una posible contradicción con las prescripciones patrimoniales, las reglas fueron adaptadas.

\section{Conclusión}

En 1969, fue realizado el primer proyecto del PPSMV dado que el Marais había devenido en ese mo- 
mento en un gran centro de la fábricas y de negocios, después de ser uno de los barrios más vetusto, insalubre, atestado y el menos aireado de la capital francesa; el PPSMV, con una idea de modernización, propuso un Plan de curetage para hacer desaparecer los problemas de higiene y salubridad. Así, propone la recuperación de las manzanas y de los patios de los inmuebles atestados de construcción para transformarlos en espacios libres con acondicionamiento vegetal, indispensables para la ventilación.

En nuestro análisis, hemos identificado que el segundo (1976) y el tercer PSMV (1980) correspondieron más bien a un reajuste de los objetivos hechos en el PPSMV (1969). No se crearon nuevos objetivos.

El cuarto PSMV (1996) es el resultado del ajuste de la salvaguardia del patrimonio, que ha actualizado los documentos gráficos y mejorado la redacción del reglamento; ha hecho un reglamento que se acerca mejor al POS (1977) de París. Desde esta perspectiva, hemos visto la creación de nuevos objetivos. Hubo un interés por flexibilizar las reglas de construcción para satisfacer la evolución, confort y seguridad del habitante y un interés por la posibilidad de construcciones nuevas.

Luego, en el 2000, la Ley SRU modificó en profundidad el derecho del urbanismo y del alojamiento en Francia. Uno de sus principales impactos fue el remplazo del POS por el PLU, más planificación y menos reglamento. El PSMV de 1996 no consideraba ciertas disposiciones del PLU ligadas al PADD y al Plan Climat. En esa perspectiva, a fin de evitar contradicciones entre el desfase de las orientaciones fijas, el PSMV (2013) armoniza el PSMV (1996) con las nuevas disposiciones provenientes del PLU. Hemos constatado también que el PSMV (2013) toma nuevas nociones ligadas a una problemática mundial, como los gases de efecto invernadero. Ciertos planes, leyes y reglamentos fueron ya impuestos. La experiencia nos muestra que damos importancia, o dicho de otro modo "ponemos en valor" lo que está en riesgo de desaparecer o que puede implicar consecuencias desastrosas. En este sentido, hemos observado que hay también una puesta en valor del patrimonio vegetal. Se trata de la inserción de nociones como: desarrollo sostenible, ecología, ambiente. Así , el PPSMV (1969) proponía el plan de curetage, un proyecto que proponía la reconquista progresiva en el centro de las manzanas o en los patios de los inmuebles de la utilización del suelo destinada al acondicionamiento vegetal. Era el mejoramiento de la calidad de vida a través de la ventilación de la ciudad. Sin embargo, hoy el PSMV (2013) toma las disposiciones del PADD y del PLU para mejorar sosteniblemente el marco de vida cotidiano de todos los parisinos promoviendo la proyección de París y estimula la creación del empleo para todos; implicar a todos los socios, actores de la ejecución del proyecto, y reducir las desigualdades para lograr un París solidario con acciones para disminuir la emisión del gas de efecto invernadero. Por consiguiente, el reglamento propone la integración de nuevas disposiciones que autorizan la colocación de dispositivos técnicos que deben economizar la energía o producir la energía renovable en las construcciones. Estas acciones conciernen, por ejemplo, a la renovación de los edificios, las energías renovables, los desplazamientos, las gestiones de los desechos, etc.

En resumen, hemos constatado que el proyecto del PSMV fue el resultado de un proceso lento de definición y adaptación sucesiva. A partir del balance de la literatura, podemos afirmar que el PSMV (2013) elaboró rectificaciones legales de gran eficacia. Los procesos de revisión y los estudios permanentes han permitido producir un documento que supera las disposiciones de contradicción aparecidos desde ciertos años. Este vuelve al proyecto un documento que funciona y que se adapta a las necesidades reales de los que lo utilizan. Así, el PSMV (2013) no solamente ha explicado bien la compatibilidad con otros documentos de urbanismo y la justificación de nuevas reglas, sino que también ha actualizado los antiguos objetivos y aportado nuevos. Este ha conocido profundas evoluciones en el transcurso de estos últimos años, como resultado tanto de la política urbana parisina como la política patrimonial del Estado. Más que los aspectos de la protección, son hoy en día los de la puesta en valor patrimonial quienes aportan los grandes desafíos del PSMV. Hemos visto igualmente que la "mirada" sobre el patrimonio se ha transformado.

En un inicio los monumentos históricos y los sectores salvaguardados fueron percibidos desde el solo ángulo patrimonial. Sin embargo, paralelamente a los documentos de planificación urbana han igualmente evolucionado en sus preocupaciones. Hoy, el PSMV muestra una visión más hibrida que integra a la vez la salvaguardia del patrimonio y la planificación urbana. 


\title{
Bibliografía
}

Atelier d'Architecture et d'Urbanisme Elisabeth Blanc et Daniel Duché. (2009). PSMV de Paris le Marais. Direction Régionale des Affaires Culturelles d'Île de France ville de Paris.

Atelier d'Architecture et d'Urbanisme Elisabeth Blanc et Daniel Duché. (2013). PSMV de Paris le Marais. Direction Régionale des Affaires Culturelles d'Île de France ville de Paris.

Ausseur Dolléans, C. (2000). Les secteurs sauvegardés. Renne, France : (s.n).

Blancot, C. (2004). PSMV du Marais. Difficultés de gestion et d'application. Amélioration et modernisation nécessaires. Paris, Frances : Atelier Parisien d'Urbanisme (APUR).

Bonnermeier A. (s.f) Le Marais - Histoire d'un vieux quartier parisien.

Collectif. (1999) Le Marais et ses abords : plan perspectif / Sauvegarde et mise en valeur du Paris historique, Paris, France.

Kehren J-N, Mahammodally N, Schneider C. (s.f) La Sauvegarde et la Mise en Valeur du Marais. Paris, France.

Mairie de Paris, direction d'urbanisme (2009). Révision du Plan de Sauvegarde et de Mise en Valeur du Marais. Paris, France.

Ministère de l'équipement, du transport et du tourisme. Préfecture de Paris (1994). Plan de Sauvegarde et de Mise en Valeur du Marais. Rapport de présentation. Paris, France.

Paris Projet (Ed.). (1970). Le plan de sauvegarde du Marais. Paris Projet. Aménagement uranisme avenir, 2, 42-74.

Pourtaud, D., Hidalfo, A. (2012). Révision du Plan de Sauvegarde et de Mise en Valeur du Marais - Bilan de la concertation.Paris, France.

Saffache P. (2005). Glossaire de l'aménagement et du développement local. Cahors, France.

\section{ANALYSE CRITIQUE DU PLAN DE SAUVEGARDE ET DE MISE EN VALEUR DU MARAIS AU TRAVERS DE SON ÉVOLUTION ${ }^{1}$}

\author{
ANDRÉ DEL / ANGIE SHIMABUKURO SHIMABUKURO
}

\section{RESUMÉ}

C’est article a été rédigé à partir d’un travail de recherche en Master2. France possède de zones urbaines dénommées secteurs sauvegardé. La possibilité de créer et délimiter ces zones a été institue à partir de la promulgation de la Loi Malraux, en 1962. Ces zones sont soumises par de règles particulières en fonction à un Plan de Sauvegarde et de Mise en Valeur (PSMV). La recherche réalisée présent l'évolution des projets du PSMV du Marais à partir de 1962 jusqu'au 2013. Lanalyse met en évidence le résultat d'un processus lent de définition et d’adaptations successives aux règlementations implémentés. Un constant décalage entre les objectifs théorique-pratique définit depuis le point de vue de la réglementation et de l'application.

MOTS CLÉS: Secteur sauvegardé, mise en valeur, patrimoine, Marais.

1. C'est article a été rédigé à partir du Memone elbaroré par Angie Shimabukuro, soutenu à Paris, le 18 Juin 2013 , avec une mention trés bien, en Master 2 recherche en "Histoire et Civilisation comparées, spécialité Ville, Architecture et Patrimoine" à l'Université Paris 7 Diderot et ENSAPVS; sous la direction de Messieurs André Del et Philippe Rivoirard. 


\section{InTROdUCtion : ConTEXTE LOCAL ET JURIDIQUE}

Paris, par la densité de son patrimoine monumental, a bénéficié des effets des lois de protection du patrimoine bâti dès leurs mises en place respectives. Il existe deux secteurs sauvegardés parisien : le Marais et une grande partie du 7ème arrondissement. Ils ont tous les deux bénéficiés d'une protection spécifique régie par un Plan de Sauvegarde et de Mise en Valeur (PSMV). Le Marais, quartier historique situé entre les $3^{\text {ème }}$ et $4^{\text {ème }}$ arrondissements de Paris, est délimité à l'Ouest par la rue Beaubourg, à l'Est par le boulevard Beaumarchais, au Nord par la rue de Bretagne et au Sud par la Seine. Voir Figure 1 - Carte du Secteur Sauvegardé du Marais. En 1964 -après la loi Malraux du 1962-, un secteur sauvegardé de 126 hectares était crée, couvrant l'ensemble du quartier du Marais. Il était à la fois le premier à être défini dans Paris et le plus vaste de France. Le Marais possède un patrimoine architectural exceptionnel de Paris et une grande homogénéité tant architecturale qu'urbaine. Le réseau des voies se trouve presque fixé avant la fin du XIIIe siècle ${ }^{1}$, les axes commerciaux ${ }^{2}$ de la ville mettent en réseau les lieux d'échanges en favorisant les déplacements et la concentration des activités économiques. Nous allons montrer ci-dessous le processus de formation historique du quartier.

\section{Situation historique du Marais.}

L’enceinte de Philippe Auguste ${ }^{3}$, qui a été construite entre 1190 et 1210 pour protéger Paris, inclut déjà la partie Nord du Marais. En 1240, l'ordre du Temple, alors très riche et puissant, construit un prieuré entouré de murailles à l'extérieur de l'enceinte de Philippe Auguste.

$\mathrm{Au}$ début du XIIIème siècle, les premières communautés religieuses s'installaient dans le Marais. Les rois français demandaient aux ordres religieux de ne plus construire de nouvelles églises et chapelles sur l'̂̂le de la Cité et leur indiquaient le Marais.

Au XIVème siècle, le Marais commence à devenir un quartier pour les nobles. Le roi Charles $\mathrm{V}^{4}$ faisait construire une nouvelle enceinte qui était annexée à la ville et qui inclut tout le Marais. Pendant la guerre de cent ans, Charles V

1. «En 1705, le réseau viaire est presque identique à celui de 1292 et en 1830 le plan de Paris n'offre que des variations infimes, produites par quelques transformations très locales (création de marché par exemple)» (Paris Projet, 1970, p.52 - p.53).

2. Ces axes correspondent aux actuelles rues Saint Martin, du Temple, Vieille du Temple et Saint Antoine.

3. Philippe Auguste ou Philippe II (1165-1223). Le règne de Philippe Auguste est une période de vives améliorations de Paris. En 1187 , il fait débuter la construction d'un mur d'enceinte sur la rive droite.

4. Charles V «Charles le Sage » (1338-1380) a été roi de France de 1364 à 1380. devait abandonner le séjour au Palais de la Cité, donc il trouve une nouvelle résidence à l'hôtel Royal Saint-Paul dans le Marais. Ensuite, le roi Charles V préféra l'hôtel Royal des Tournelles ${ }^{5}$, cet hôtel reste résidence parisienne des rois français pendant cent cinquante ans jusquà la mort du roi Henri $\mathrm{II}^{6}$. Peu à peu, à partir de 1358 , commence à s'installer la noblesse et les classes plus riches en édifiant leurs demeures auprès du roi.

Du XVIème au XVIIème siècle, se produit l'apogée du quartier où la densité des habitations bourgeoises augmen$\mathrm{te}^{7}$ et la Place Royale ${ }^{8}$ - actuelle Place des Vosges - devient le centre du Paris élégant. ${ }^{9}$ Pendant l'apogée du Marais, de nouvelles communautés religieuses apparurent ${ }^{10}$.

A partir du début du XVIIIème siècle, la noblesse commence peu à peu à quitter le Marais - en déménageant vers l'Ouest- et les artisans remplacent la noblesse. Après l'ère de mondanité qui caractérise l'époque d'urbanisation du Marais sous Henri $\mathrm{IV}^{11}$, le quartier se teinte d'austérité. Le Marais devient peu à peu un "vieux quartier » et commence alors une ère de saturation et de perte de vitesse. De là, l’apogée du Marais s’effrite ; un lent déclin commence. « Leurs églises et bâtiments étaient ou bien transformées en magasins ou bien détruits. Les artisans et les ouvrières des quartiers voisins prenaient possession des grands hôtels désertés». (Bonnermeier, p.14).

L’empereur Napoléon Ier ${ }^{12}$ avait quelques idées pour remodeler le centre de Paris, il a fait construire des greniers de

\footnotetext{
5. Construit en 1417 et situé où se trouve la Place de Vosges.

6. Henri II (1519-1559) est roi de France de 1547 à 1559.
}

7. L'hôtel de Sully (1624), I'hôtel de Mayenne (1613), I'hôtel Carnavalet (1544), I'hôtel de Lamoignon (1585) et I'hôtel Salé (1656). La dernière réalisation prestigieuse du Marais était celle de l'hôtel de Rohan-Soubise (1705) pour le Duc de Rohan. (Bonnermeier, p.12p.13).

8. La place a été inaugurée en 1612 à l'occasion des mariages de Louis XIII avec I'Infante Anne d'Autriche. Dans le pavillon N6 a notamment vécu Victor Hugo, entre 1832 et 1848.

9. Après la mort de Henri II, la reine Catherine de Médicis renonce à habiter les Tournelles et elle fait construire le Palais des Tuileries. Toutefois, le Marais reste quand même un quartier aristocratique. Elle ensuite décidé de faire démolir l'hôtel Royal des Tournelles. Le roi Henri VI reprend alors I'idée de Charles IX en ordonnant en 1605 l'aménagement d'une grande place bâtie de quatre côtés d'une même symétrie. "L'ensemble de la Place Royale fait à la fois impression élégante et pratique, harmonieuse et claire. Elle devenait au XVIIème siècle le centre du Paris élégant, représentant une sorte de cœur du Marais» (Bonnermeier, p.11).

10. Les Minimes, les Capucines (1524), les Filles de Saint-Elisabeth (1626) et les Jésuites.

11. Henri IV (1553-1610) a été roi de Navarre de 1572 à 1610) puis roi de France de 1589 à 1610 . Il est le premier souverain français de la branche dit de Bourbon de la dynastie capétienne.

12. Napoléon ler (1769-1821) a été sous le nom de Napoléon Bonaparte, un général des armées de la Révolution. 
réserve dans la partie Sud-ouest du Marais et dans les années 1830 les quais de la Grève et de l'hôtel de Ville furent entièrement reconstruits et élargis. Ensuite, sous Napoléon III $^{13}$, le centre de Paris a été de nouveau remodelé grâce au Baron de Haussmann ${ }^{14}$, beaucoup d'anciens bâtiments ont disparu; toutefois, une grande partie du Marais est restée telle quelle, comme un quartier pauvre et sans intérêt.

$\mathrm{Au}$ XIXème siècle, le Marais devenait un quartier ouvrier, sale, insalubre et en mauvais état. Jusqu'après la deuxième guerre mondiale (1939-1945) l'état sanitaire de la population était le plus mauvais. Pas seulement des nombreux hôtels furent démolis pour construire à leur place des immeubles avec plus détages, mais des entreprises industrielles et commerciales s'installaient dans le quartier.

Quelques années après de la deuxième guerre mondiale, le Marais est resté le quartier le plus sale de Paris et en très mauvais état. En 1962, la «Loi Malraux» sauve le quartier. Andreas Bonnermeier indique que:

Pour pouvoir sauver le Marais, il était nécessaire de supprimer l'industrie et le grand commerce, c'est-à-dire 'refondre tout le quartier pour en faire une zone résidentielle'. Le Marais, de cette façon devenue un quartier de plus en plus recherché, entrainait le départ de nombreux ateliers et entrepôts 'étroitement imbriqués aux logements', mais il se développait un commerce de détail avec des cafés, des restaurants, des boutiques de cadeaux, etc. Certains métiers traditionnels se maintenaient, conservant au Marais une réelle importance économique pour les activités de la bijouterie-joaillerie, de la lunetterie et des instruments d’optique, des 'métiers d'arts', de la maroquinerie et de la confection. (p.19.)

Finalement, en 1990, le Marais se restaure avec d'innombrables chantiers; de plus, beaucoup de musées ont pris leur siège dans des bâtiments anciens du quartier ${ }^{15}$. «De plus en plus de gens cherchent un logement dans ce quartier, car il est 'à la mode' d'habiter le Marais. Le Marais est alors redevenu un quartier chic et 'en vogue', mais aussi cher (quant aux loyers).» (Bonnermeier. p.22.)

\section{De la protection de monument à la sauvegarde de quartiers historiques}

13. Napoléon Illème (1808-1873) est le premier président de la République française, élu le 10 décembre 1848.

14. Georges-Eugène Haussmann (1809-1891) a été préfet de la Seine du 1853 au 1870. Il a dirigé les transformations de Paris.

15. L'hôtel Aubert de Fontenay (le musée Picasso), l'hôtel de Lamoignon (la bibliothèque historique de Paris), I'hôtel Guénégaud (le musée de la Chasse et de la Nature), I'hôtel de Soubise (le musée de l'histoire de France et l'Archive national), I'hôtel Carnavalet (où habitait de 1677 à 1696 la Marquise de Sévigné, se trouve un musée avec des souvenirs de la Marquise, des meubles et des témoins des différents époques).
Chantal Ausseur Dolléans (2000) explique que :

Pour nombre de théoriciens et de praticiens de cette époque, le patrimoine bâti se limitait aux monuments, dont la protection était assurée par la loi du 31 décembre 1913 relative aux monuments historiques, étendue par la loi du 25 février 1943 à leurs abords. (p.12)|

La «loi du 31 décembre 1913» définit les critères de classement, les intervenants obligatoires, etc. Elle visait la protection des monuments historiques eux-mêmes, et non de ce qui les entoure. Puis, la «Loi du 25 février 1943» modifie la loi du 1913, c'est la naissance de la notion « d'abord de monuments historiques». Elle impose une forme de vigilance à l'égard des projets de travaux dans le «champ de visibilité» de 500 mètres du monument historique dans lequel l'Architecte des Bâtiments de France (ABF) doit délivrer un avis de conformité.

Après la Deuxième Guerre Mondiale, à la fin des années 50, un grand parti de quartiers et des centres historiques ont été en état de dégradation et insalubrité. Ausseur (2000) trouve que :

La tentation était grande d’appliquer à ces centres urbains fragilisés les solutions radicales prônées par les tenants de l'idéologie hygiéniste : démolir et reconstruire selon les principes du zonage testé alors sur les quartiers périphériques. La ville existante, avec ses quartiers par essence confinés où les vides et les pleins sont étroitement imbriqués, semblait en effet bien en peine de répondre, à ces valeurs exclusives de salubrité, d'espace et de circulation. (p.14)

Durant les années 60 s'est produit nombreuse aménagements de centres urbains. André Malraux ${ }^{16}$ prend conscience de l'importance de la viabilité urbaine par rapport à l'intégralité des quartiers historiques de la ville et - suite à l'expérience du périmètre de restauration immobilière de Tours en 1961/1962- il promulgue la loi Malraux ou loi $n^{\circ}$ 62-903 du 4 août 1962, la quelle institué la possibilité de création et délimitation des secteurs sauvegardes pour parte de l'État. Malraux fait un discours et propose « de sauver lâme des cités et de changer le visage de la France », il explique « la notion d'ensemble urbain » où l'originalité de sa loi réside dans « la création d'un outil d’aménagement, permettant d'introduire dans les immeubles anciens, le confort moderne », et où l'objectif principal est la protection et la mise en valeur d'ensembles urbains présentant un patrimoine majeur et particulièrement dégradés. C'est la naissance de la protection à l'échelle d'un secteur urbain tout entier avec une prise de conscience lié à l'harmonie, l'unité, la cohérence et l'organisation de l’ensemble urbain.

16. George André Malraux (1901 - 1976), est un écrivain et un politique français, nommé le 08 janvier 1959 ministre d'Etat chargé des affaires culturels. 
Suite à la promulgation de la loi Malraux et à l'impact culturel et artistique que représentait le festival du Marais ${ }^{17}$, en 1964 se crée le secteur sauvegardé du Marais, le premier secteur mis à l'étude ${ }^{18}$ après l'adoption de la loi Malraux, mais il n’a néanmoins été approuvé qu’en 1996, après 32 ans d'étude.

Dans le dossier ${ }^{19}$ de la Direction Régionale des Affaires Culturels (DRAC) d'Île de France (2013), élaboré par l'Atelier Blanc-Duché, en explique que:

Cette loi a été conçue pour empêcher que se poursuive la destruction systématique des centres urbains, organisée au nom de la rénovation dans l'immédiat après-guerre. Depuis une vingtaine d'années, la notion de patrimoine même a évolué : il sagit aujourd'hui de lui rendre une fonction vivante, et non plus simplement muséale, en l'intégrant dans l'urbanisme contemporain. (p.25).

\section{Cadre de recherche}

Nous allons présenter, chronologiquement, lévolution du PSMV du Maris à travers des projets (en 1969, 1976, 1980, 1996 et 2013) et des processus de révision (en 1977, 1979 1980, 1984-1996 et 2006-2013). Voir le Graphique 1- Ligne du temps comparative (1960-2013). Par commodité du lecteur nous indiquerons les plans successifs sous la forme PSMV (xxxx), selon l’année de son élaboration.

En 1962, le Marais est devenu d'un côté le principal atelier de Paris, le grand centre de "la fabrique et du négoce ", et de l'autre, l'un des quartiers le plus vétuste et insalubre, c'est en quelque sorte le quartier le moins aéré et le plus encombré de la capitale ${ }^{20}$.

17. Le festival du Marais est un festival annuel réalisé entre 1961 et 1993. II a été créé à l'initiative d'un groupe de citoyens réunis autour de Michel Raude, à la même date que l'association pour la sauvegarde et la mise en valeur du Paris historique.

18. «Les premières études de secteurs sauvegardés ont été confiées à des Architectes en chef des monuments historiques, les seuIs à cette époque, travaillant sur le patrimoine, mais avec une vision monumentale bien éloignée des problèmes de la ville dégradée et insalubre. » (Atelier Blanc-Duché, PSMV de Paris le Marais - Rapport de présentation préambule, 2013, p.4).

19. Dossier d'enquête publique sur le « Projet de révision du Plan de Sauvegarde et de Mise en Valeur du Marais », dossier n³-partie III, justification des règles et la compatibilité avec les autres documents d'urbanisme.

20. Les espaces verts représentent $1,71 \%$ de la surface. Les espaces libres sont rares, l'occupation du sol dépasse $85 \%$ (55\% pour l'ensemble de Paris). Constructions amoncelées où l'aire et la lumière pénètrent de manière parcimonieuse. Certains îlots atteignent jusqu'à 2000 habitants/ha. Confort des logements : Les plus mauvaises de tous les arrondissements parisiens, $30 \%$ dépourvus d'eau courante, $60 \%$ sans WC, $10 \%$ sans éclairage électrique. Equipements sociaux insuffisants : $3 \mathrm{~m}^{2}$ de locaux scolaires par enfant. (Paris Projet, p.47)
Le premier projet, le Plan Permanent de Sauvegarde et de Mise en Valeur ${ }^{21}$ de France, soumis au conseil de Paris en 1969, considérait la ville comme un monument et proposait une image idéale par rapport aux habitants et aux activités. Dans ces conditions, «l'idée d'une modernisation» du Marais a précédé celle d'une restauration de cet ancien quartier. Le Plan avait une double préoccupation - tracer des axes de pénétration et élargir les voies de circulation ; et rénover des îlots (insalubres et délabres). Il visait la conservation et préservation de «l'aspect architectural traditionnel du Marais, en protégeant les constructions importantes, en favorisant la restauration immobilière et en assurant l'homogénéité de leur environnement, et à créer des conditions plus satisfaisantes pour la vie du quartier et de ses habitants» (Paris Projet, 1970, p.55).

Entre 1962 et 1971, une période interventionniste, produit "des projets de ville idéale ", devant s'imposer aux villes et aux propriétaires désireux d'intervenir sur leur patrimoine. C'est aussi lépoque des «îlots opérationnels» ${ }^{22}$ gérés par une société d'économie mixte chargée des opérations, dont le bilan normalement déficitaire était pris en charge par 1'Etat (Atelier Blanc-Duché, 2013).

Il avait été étudié «à îlot» et «à l'immeuble» et proposait l'une des premières images globales définissant à long terme le devenir d'un quartier historique en constituant un guide pour les modifications architecturales de la zone. Ensuite, à partir de 1968 commence une période d'enlisement ${ }^{23}$ qui sétendit jusqu'en 1976 .

Le PSMV (1976) passe «d'un projet utopique à un outil de gestion juridique». Il est une nouvelle version du PSMV, plus raisonnable pour faire face aux difficultés de maintien de la population, des activités ${ }^{24}$ et de relogement. Il propo-

21. On parlera parfois aussi l'appellation « Plan Permanent de Sauvegarde et de Mise en Valeur ", pour designer la même procédure et vouloir afficher sa permanence. Le nombre de mise en révision du PSMV du Marais est là pour témoigner que cette permanence est relative.

22. Sur les 60 secteurs sauvegardés mis à l'étude, 30 firent l'objet d'îlots opérationnels, dans lesquels le principe de la rénovation urbaine (démolition/reconstruction), a été souvent appliqué. » (Atelier Blanc-Duché, PSMV de Paris le Marais - Rapport de présentation préambule, 2013, p.4).

23. «Plusieurs facteurs sont à l'origine de ce phénomène. En premier lieu, une antinomie entre une loi qui prône la conservation et la mise en valeur du patrimoine bâti et des plans programmant la destruction d'îlots complets ou la création de vrais faux espaces publics historiques, comme à Troyes où l'on voulait gommer la trame viaire médiévale en créant des galeries couvertes sous les immeubles à pan de bois que l'on aurait évidé sur un niveau. Mais le facteur le plus crucial est celui du manque de moyens, avec la disparition de la politique des îlots opérationnels et des financements spécifiques. » (Atelier Blanc-Duché, PSMV de Paris le Marais - Rapport de présentation préambule, 2013, p.4 - p.5).

24. Après 1973 et le primer choc pétrolier, l'artisanat est en crise, 
se de : réduire les curetages ${ }^{25}$ et les opérations de renouvellement urbain, de supprimer les élargissements de voies encore en vigueur, remplacés dans certains cas, par des passages sous arcades dans les bâtiments existants. Ce plan conservait une part très importante de curetages, environ 1000 propriétés sur les 1900 existantes. Ces intentions de curetages ont joué un rôle majeur dans la disparition des activités existantes, puisque la réhabilitation ou la transformation des locaux était, dans de nombreux cas, interdite.

En 1977 se réalise la première révision du projet, le groupe de travail s'est prononcé pour une meilleure prise en compte des intentions d'aménagement et un assouplissement de certaines règles. Un diagnostic général du projet est réalisé, pour mettre en évidence des secteurs d'aménagement d'ensemble, des espaces verts, des cheminements piétons et l'adaptation des règles - calquées sur celles du Plan d'Occupation des Sols (POS) de Paris- aux particularités du secteur sauvegardé. ${ }^{26}$ (Atelier Blanc-Duché, 2013).

Puis, entre 1979 et 1980 se produit la deuxième révision du projet. En 1978, la ville de Paris assure un meilleur maintien des activités artisanales et industrielles que ne le prévoyait le plan. Le conseil municipal adopte une orientation majeure pour le plan de sauvegarde, qui visait à permettre le maintien des entreprises industrielles et artisanales dans le quartier, alors que le plan proposé prescrivait la démolition de nombreux locaux d'activités, et excluait leur amélioration et leur adaptation (Atelier Blanc-Duché, 2013). Il était souhaité par le groupe de travail et par les architectes du Marais que le plan fasse mieux ressortir les intentions de la collectivité (Kehren, Mahammodally et Schneider). Le plan s'est attaché: à mieux adapter les règles architecturales aux spécificités du tissu du Marais, à mieux prendre en compte des intentions d'urbanisme, à sortir du raisonnement à la parcelle, et enfin, à nuancer les curetages en mettant en place un dispositif modulé destiné à éviter la disparition systématique des activités industrielles et artisanales. Les objectifs de la révision des curetages ont été le dégagement des abords des monuments historiques et des ensembles urbains majeurs, et la création de jardins en cœur d'îlots qui ont été abandonnés ou différés, afin de permettre le maintien d'activités existantes ou la réhabilitation de logement.

la désindustrialisation textile et de la confection est amorcée, les métiers du métal et du meuble sont en difficulté, la grande mutation du Marais est amorcée et sera dès lors, inéluctable.

25. Opération de réhabilitation caractérisée par une destruction des constructions établies dans les espaces intérieurs. (Saffache, 2005, p. 71)

26.Notons, par exemple à ce sujet, le gabarit des constructions futures et la possibilité de transformation à l'occasion de réhabilitation (Atelier Blanc-Duché, PSMV de Paris le Marais - Rapport de présentation préambule, 2013, p.13).
Le PSMV (1980) approuvé par le Conseil de Paris ${ }^{27}$ le 22 Septembre 1980, où «les modifications ont surtout concerné les curetages, les règles d'architecture et les intentions d'urbanisme» (Kehren, Mahammodally et Schneider, p.23.)

En 1980 se produit l'approbation du document de réexamen complet du plan ${ }^{28}$. De nouvelles mises au point on été ensuite réalisées en raison des évolutions du tissu urbain et de l'étendues du secteur. Deux types d’opérations ont modifié la structure urbaine du Marais au XIXème siècle. D’une part les percées de voies nouvelles et les définitions d'alignements, et d'autre part les opérations isolées. Entre 1984 et 1996 se réalise une troisième révision du projet, où après plusieurs années d'études et d'analyse du quartier, l'architecte Bernard Wagon est missionné, en 1984, pour mener à bien lélaboration de la révision du PSMV. Ce PSMV a été rendu public en 1992, mis à l'enquête publique en 1994 et approuvé par décret du 23 août 1996.

Le PSMV (1996), est le résultat d'un ajustement de la sauvegarde du patrimoine, présente depuis de la loi Malraux et les premières études du PSMV, et de l'information exprimée dans le rapport d'enquête publique de 1994, de «l'attention des résidents du Marais pour la valeur historique, architectural et urbaine du quartier et du souhait de la Ville de Paris de maintenir le caractère évolutif des lieux de leurs les activités». En ce qui concerne nouvelles dispositions, le projet propose l'introduction de « sous-secteur d'aménagement d'ensemble» au plan.- leur particularité est reporté sur plan sous la forme de périmètre d'aménagements dotés d'une marge réglementaire plus large en matière de construction neuves- et la séparation de deux situations particulières spécifiques, d'un part la protection, la mise en valeur et la restauration du patrimoine architectural des bâtiments existants ${ }^{29}$ et d'autre part les possibilités de constructions nouvelles, extensions et modifications relatives à la volumétrie et à l'implantation, en présentant une meilleure ouverture vers l'architecture contemporaine. Ainsi, des ajustements se sont traduits par :

La mis à jour des documents graphiques, en particulier en ce qui concerne les opérations réalisées de curetages ou constructions (Atelier Blanc-Duché, 1996).

27. «C'est sur la base des objectifs nouveaux que l'équipe des architectes du plan de sauvegarde du Marais, I'APUR, et la Direction d'aménagement et de l'Urbanisme de la Ville de Paris, en liaison avec les services de l'Architecte des Bâtiments de France, ont pu procéder à un réexamen complet du projet du plan. » (Jean-Nicolas Kehren, Nourani Mahammodally, Christine Schneider. La Sauvegarde et la Mise en Valeur du Marais, p.23.)

28. Réalisé par un groupe de travail composé des architectes du PSMV associés à l'Atelier parisien d'urbanisme, la Direction de l'aménagement urbain de la ville de Paris et les architectes de bâtiments de France.

29. II permet toutes initiatives visant à restituer les dispositions historiquement conformes pour les édifices anciens. 
Les curetages maintenus sont des immeubles dont la démolition pourra être imposée à l’occasion d’opérations d’aménagement publiques ou privés.

La prescription de «façade à conserver » a été étendue aux certains axes principaux ${ }^{30}$. La conservation en raison de leur intérêt architectural, pittoresque ou de leur caractère d'édifices d'accompagnement.

Le réexamen de certains articles du règlement qui à permis une amélioration de la présentation de la rédaction du document et un ajustement de certaines dispositions à la lumière de l'expérience acquise. (Ibid.)

Amélioration de la rédaction du règlement quant aux généralités, aux définitions et à l'instruction des dossiers. (Ibid.)

Amélioration de la définition des espaces libres et des règles qui leur sont affectées. Introduction des espaces protégé au titre des " cours pavées » au document graphique, afin d'avoir une harmonie urbaine de cour d'îlots. (Ibid.)

L'ensemble du dispositif de protection et de mise en valeur est maintenu ; toutefois un assouplissement des règles de constructibilité a été introduit pour satisfaire l'évolution de l'habitabilité, le conforte et la sécurité. (Ibid.)

L'introduction au plan de prescriptions visant à protéger les perspectives urbaines du XIXème siècle. (Ibid.)

${ }^{31}$ La plupart des immeubles intéressants à ce titre sont datés de l'époque comprise entre 1850 et 1930.

Après la création du PSMV (1996) sont apparues certaines lois, notamment la loi relative à la Solidarité et au Renouvellement Urbains (SRU) du 13 décembre 2000 qui prévoit la disparition du Plan d'Occupation du Sol (POS) ${ }^{32}$ de 1977 et institue les Plan Local d'Urbanisme (PLU) ${ }^{33}$ de

30. La rue Rivoli, la rue François Miron, la rue des Archives (en partie) et, en périphérie du secteur, au boulevard Henri IV', dont les immeubles présentent une richesse architecturale exceptionnelle.

31. «La protection des perspectives constituées par les immeubles du XIXème siècle a été notablement étendue, dans l'objectif de maintenir la cohérence des façades. La protection de ces édifices est limitée aux façades uniquement, et vise à interdire l'altération de l'unité assurée par l'échelle des immeubles et la modénature qui les relie les une aux autres» (Atelier Blanc-Duché, PSMV de Paris le Marais - Rapport de présentation préambule, 1996, p.13.)

32. Un document d'urbanisme dont le régime a été crée par la Loi d'orientation foncière de 1967. Le POS subsistent et gardent toute leur validité juridique tant qu'ils n'ont pas été transformés en PLU.

33. Un document stratégique en même temps qu'un outil réglementaire. II comprend un rapport de présentation du contexte et des perspectives d'évolution de la ville, un Projet d'Aménagement et de Développement Durable (PADD) qui définit les orientations d'urbanisme et d'aménagement pour tout le territoire, un règlement qui fixe les règles d'utilisation des sols illustrées par des documents graphiques (cartes, plans, schémas) et des documents juin 2006. Elle place l'urbanisme au centre des réflexions portant sur le territoire et ainsi les secteurs sauvegardés s'inscrivent dans la logique urbaine d'ensembles beaucoup plus large. Cette réforme du droit de l'urbanisme a ouvert la nécessité d’analyser la compatibilité du PSMV avec les autres documents d'urbanisme. Par ailleurs, le contexte économique a évolué depuis les années 1960 ; si bien que, le Conseil de Paris, lors de sa séance des 24 et 25 juin 2002 demande à l'Etat la mise en révision des PSMV du Marais et du $7^{\text {ème }}$ arrondissement (Blancot, 2004). Ensuite, ce conseil, lors de sa séance du 27 janvier 2003 a examiné le document d'orientation pour l'élaboration du Projet d’Aménagement et de Développement Durable (PADD) inclus dans le PLU-, il indique que : «Les deux territoires couverts par les plans de Sauvegarde et de mise en valeur du Marais et du $7^{\text {ème }}$ arrondissement sont concernés par les nouvelles orientations définies dans le PADD. Au-delà de la nécessaire préservation d'un patrimoine exceptionnel, des dispositions doivent être prise pour renforcer le dynamisme de ces quartiers, permettre à leur habitants de s'y maintenir et enrayer ainsi les phénomènes de muséification» (Blancot, 2004, p.1). Ainsi, un «Bilan sur les difficultés de gestion et d'application. Amélioration et modernisations nécessaires» a été fait d’octobre 2003 à février 2004 par l'Atelier Parisien d'Urbanisme (APUR). Létude souligne qu'il est d'une part urgent de compléter le PSMV pour l'harmoniser avec le PADD et le futur PLU de Paris, et d'autre part elle préconise que soient lancées, dans le même temps, les études urbaines et patrimoniales indispensables pour mettre à jour le PSMV et se prononcer sur son extension.

Puis, la révision du PSMV a été engagée par arrêté ministériel du 15 juin 2006, suivie par la Commission Locale du Secteur Sauvegardé (CLSS), créée par arrêté préfectoral du 19 février 2009 et composée du Maire de Paris et du Préfet, de conseillers de Paris, de représentants des services de l'État et de personnalités qualifiées. Cette commission a validé les travaux détudes, le projet du PSMV et a proposé le «cabinet d'architectes-urbanistes l'atelier Blanc et Duché associant des compétences en matière d'histoire, de patrimoine et d'économie, [...] pour conduire les études nécessaires à la révision du PSMV [...]» (Mairie de Paris, 2013).

En mai 2009, un document provisoire -une phase d'étude qui montre une première avance du document final- de la révision du PSMV du Marais est présentée par l'atelier Blanc et Duché. Les groupes de travail ont pour but d'alimenter la réflexion, en faisant remonter un maximum d'information, en auditionnant des spécialistes sur différents sujets tels que : l'harmonisation PLU-PSMV, l'espace public, les cours intérieures et le développement durable.

techniques annexes, ». (Mairie de Paris, Direction de l'Urbanisme, Op.cit, p.4) 
Ensuite, entre le 2009 et le 2010 se réalise une " concertation des 3ème et 4éme arrondissements ", tout au long de lélaboration du document : réunions publiques, ateliers thématiques, conférences, etc., pour prendre connaissance des attentes de chacun et recueillir les différents avis. Le résultat du travail participatif est un «Bilan des réunions publiques de concertation préalable légale » réalisé le 05 novembre 2012, qui a pour but de forger des propositions pour le PSMV dans trois secteurs différents : Patrimoine, préoccupation pour protéger de nombreuses constructions du XIXème et XXème siècle, et le nombre des prescriptions de démolition ont diminué. Développement Durable, avec le prolongement de la loi Grenelle II se produit un changement sur la superficie minimale de pleine terre et les règles d'hauteur de construction pour l'installation de dispositifs d'énergie renouvelable. Fonctions urbaines (logements, équipements, commerces) la mixité sociale sera imposé un pourcentage de logements sociaux et la protection et encouragement du commerce et de l'artisanat (rôle d'animation et de vie du quartier).

Une enquête publique a été réalisée dans les mairies des 3e et 4e arrondissements, du 18 février au 22 mars 2013, il tiendra compte des éléments issus de la concertation validé par la CLSS après avis de la Commission Nationale des Secteurs Sauvegardés (CNSS). Pendant l'enquête, le public a pris connaissance du dossier et consigné ses observations, propositions et contre-propositions sur le registre, qui était à disposition, lors de la consultation du dossier d'enquête.

Finalement, le PSMV (2013) a été approuvé par arrêté du Préfet de la région d'Île-de-France -Préfet de Paris- le 18 décembre 2013. Il réponde à une meilleure connaissance et protection du patrimoine. Il s'adapte mieux aux typologies historiques des bâtiments protégés, afin de mettre en valeur et contribuer à : l'équilibre de habitat/emploi et mixité sociale, à la qualité de vie des quartiers et à la préservation de lenvironnement des orientations du PADD.

\section{État de la question: Déroulement de la recherche}

Nous avons trouvé un bon matériel de travaux, précis et fouillé. Les informations concernant le Marais sont riches, et s'intensifient jours après jours. En ce qui concerne à l'information du PPSMV de France, nous avons pris comme point de départ les archives privées de la Médiathèque de l'Architecture et de l'Patrimoine, où se trouvent tous les dossiers des études opérationnelle pour le PPSMV en 1968. Nous avons consulté aussi la Bibliothèque du Pavillon de l'Arsenal- où nous avons tiré le contenu notamment de la revue bimestrielle «PARIS PROJET. Aménagement Urbanisme Avenir » 2e trimestre 1970, un article préparé à partir du rapport établi par léquipe des architec- tes Louis Arretche ${ }^{34}$, Michel Marot ${ }^{35}$ et Bernard Vitry, assistés de Maurice Minost ; chargés de l'élaboration du Plan du Marais et du Mémoire présenté par le Préfet de Paris à l'Assemblée Municipale en octobre 1969.

Nous avons consulté aussi la Bibliothèque nationale de France $(\mathrm{BnF})$, la Bibliothèque Cité de l'Architecture et du Patrimoine (Palais de Chaillot), le Centre de Ressources Documentaires Aménagement, Logement, Nature (CRDALN) - ex Centre de Documentation de l'Urbanisme (CDU), l'Association pour la Sauvegarde et la Mise en Valeur du Paris Historique (ASMVPH), l'École Nationale Supérieur d'Architecture Paris Val de Seine (ENSAPVS), l'Université Paris Diderot - Paris 7 et la Bibliothèque Historique de la Ville de Paris (BHVP).

Finalement, cette recherche a été soutenue le 18 juin 2013; donc, malgré le fait que le PSMV (2013) a été approuvé le 18 décembre 2013, notre recherche s'arrête jusqu'à l'enquête publique réalisée dans les Mairies des $3 \mathrm{e}$ et $4 \mathrm{e}$ arrondissements de Paris, du 18 février au 22 mars 2013. Ainsi, nous avons utilisé un PSMV qui était encore en révision et qui n'a pas pris en considération le rapport du commissaire enquêteur.

\section{Méthodologie}

Lanalyse du PSMV montre un constant décalage entre les objectifs définis par le cadre juridique et les applications. A cet égard, dans le cadre de l'étude de la politique de protection et de la valorisation du patrimoine, nous avons analysé tous les aspects concernant à l'évolution du PSMV, afin d'approfondir et d'élargir la portée dans un nouveau document et essayer de trouver une réponse à la préoccupation issue de cette évolution. De ce fait et à partir de cette procédure d'évolution, nous nous posons la question. Comment ces objectifs et règlements ont-ils évolués ? Nous avons constaté qu’au cours de ces quarante dernières années, la zone couverte par le PSMV a connu de profondes évolutions. Il est aujourd'hui impensable de réaliser un PSMV sans tenir compte de ses effets sur la dynamique urbaine du territoire en question. Donc, cette analyse a pour but de mettre en évidence : Lévolution historique du quartier du Marais. Lévolution de la procédure de protection générale, c'est-à-dire, l'évolution de la procédure de protection du patrimoine au niveau des documents juridiques de la ville de Paris. Lévolution de la procédure de protection spécifique, c'est-à-dire lévolution de la procédure des projets du PSMV, en montrant la problématique et la proposition de chaque projet du PSMV, et les nombreuses et constantes études de révision du PSMV dès le début jusqu’à nos jours, en montrant les témoins de l'évolution de la théorie à la pratique. Lanalyse critique et comparative des documents graphiques et documents texte du PSMV. Une méthode

34. Louis Gerald Arretche (1905-1991) est une architecte et urbaniste français.

35. Michel Marot (1926) est une architecte français. 
d'analyse graphique réalisée en créant une série de cadres synoptiques, afin de faciliter la compréhension du sujet. Il fournit une structure globale et cohérente du sujet de ses nombreuses relations.

\section{Résultats}

Le PSMV a considérablement évolué. Afin de faire une analyse claire et concise, nous avons trouvé opportun de faire une analyse en trois parties : En premier lieu, les objectifs initiaux du premier projet de PSMV (1969). En deuxième lieu, lévolution des objectifs des différents projets du PSMV (1969, 1976, 1980, 1996 et 2013). Et en troisième lieu, les objectifs du PSMV (2013), en faisant une comparaison plus précise avec les documents textes (réglementaires) et de documents graphiques (cartes) du PSMV (1996). Voir Graphique 2 - Diagramme l'évolution des objectifs du PSMV.

\section{Les objectifs initiaux du premier projet PSMV de 1969:}

Afin de donner une solution à la problématique de salubrité et hygiène du Marais, le PSMV a retenu certains objectifs depuis ses origines.

Nous avons identifié et énuméré cinq objectifs qui sont liés entre eux-mêmes. Voir les cases de couleur jaune dans le Graphique 2 - Diagramme l'évolution des objectifs du PSMV

Objectif 1: Conservation de l'architecture traditionnelle et assurance l'homogénéité. Nécessité de savoir identifier les bâtiments à protéger et les bâtiments à démolir.

Objectif 2: Plan de curetage. C'est-à-dire, une opération de réhabilitation urbaine qui a pour objectif la restauration d`un îlot urbain ancien (selon laquelle on détruit un immeuble sauf la façade quand elle présente un intérêt historique ou architectural et que l'on reconstruit intégralement l'arrière de l'immeuble), une intervention portant sur lhygiène, le confort et l`authenticité esthétique.

Objectif 3: Réanimation à travers des fonctions nouvelles : compenser les fonctions disparues par les démolitions, afin de donner une nouvelle activité aux bâtiments conservé.

Objectif 4: Créer des conditions plus satisfaisantes pour la vie du quartier et de ses habitants.

- Objectif 5: Tracer des axes de pénétration et élargir les voies de circulation.

\section{Lévolution des objectifs des cinq projets du PSMV (1969, 1976, 1980, 1996 et 2013):}

Il correspond à lévolution de tous les projets de PSMV. Au fur et à mesure de notre analyse, nous avons constaté qu'il avait trois types d’objectifs : adaptés, arrêtés (objectifs encore existants, mais qui non pas évolué) et ajoutés (objectifs qui ont apparu après le premier projet).

\subsection{Les objectifs adaptés}

Nous avons vu que des cinq objectifs proposés dans le premier projet, trois ont évolué jusqu'au PSMV (2013).

Dans cette perspective, nous allons présenter chaque évolution selon la numération que nous avons proposée ci-dessus.

Conservation de l'architecture traditionnelle et assurance l'homogénéité (objectif 1). Nous avons observé que l'originalité notable du Plan a été de conserver laspect architectural traditionnel du Marais, en protégeant les constructions importantes et en assurant l'homogénéité de leur environnement. La légende du premier document graphique, concernant aux bâtiments à conservé ou protégé ont été catégorisé de la manière suivant : Immeuble protégé au titre des Monument Historique $(\mathrm{MH})$, immeuble intéressant à protéger, immeuble d'accompagnement à conserver et façade particulièrement intéressante. Cependant, commence à apparaitre la nécessité d'adapter les règles architecturales au tissu urbain. De sorte que, le PSMV (1980) met à jour les règlements, propose des adaptations des ces règles d'architecture. Après, le PSMV (1996) étend la protection aux immeubles du XIXème siècle et les façades passent dêtre «façade particulièrement intéressante» à être «façade à conserver». Enfin, le PSMV (2013) a l'objectif de hiérarchiser la liste de bâtiments protégés sans délimitation de période, de faire évoluer les règles de protections et de restauration pour conserver la mémoire des transformations urbaines, et de respecter la structure du tissu urbain du découpage parcellaire existant.

Plan de curetage (Objectif 2). Compte tenu du problème de la densité d'occupation du sol, le $1^{\text {er }}$ projet, le PSMV (1969) propose un plan de curetage et de dégagement du centre des îlots. C’était un projet qui proposait de reconquérir progressivement, dans le cœur des îlots ou dans le cours des immeubles les espaces libres indispensables à l'aération du Marais. Si bien que, le PSMV (1969) a proposé des bâtiments dont la démolition est souhaitable ou certaine, afin de récupérer le sol pour prioriser l'aménagement végétal avec des «jardins continus» crée au cour des îlots et ouverts au public. Néanmoins, la quantité de curetage a été utopique; de sorte que le PSMV (1976) réduit les curetages environ 1000 propriétés sur les 1900 existantes. Afin d'éviter des problèmes de disparition systématique des activi- 
tés industrielles et artisanales; le PSMV (1980) fait des modifications concernant aux curetages, aux règles d'architecture et aux intentions d'urbanisme. Ensuite en 1996 le curetage change d'être «Démolition souhaitable» et «Démolition certaine» à être «Immeuble dont la démolition pourra être imposée à des fins de mise en valeur ou en raison de leur vétusté» et «immeuble dont la démolition pourra être imposée sauf en cas de maintien des activités industrielles, artisanales ou commerciales». Finalement, dans le PSMV (2013) est proposé revoir la liste des bâtiments voués à la démolition.

Tracer des axes de pénétration et élargir les voies de circulation (Objectif 5). La circulation est un aspect important pour le bon développement de la ville. Au début il a été imposé une politique favorable à l'automobile qui a été suivie jusqu'au PSMV (1996). Cependant, aujourd'hui, c'est le PADD qui propose une politique de déplacement. Dans cette perspective, le PSMV (2013) propose réduire les possibilités de stationnement pour participer à la politique des déplacements mise en œuvre à l'échelle de Paris.

\subsection{Les objectifs où l’évolution est été arrêtée}

Il s'agit des objectifs existant, mais qui n'ont pas eu la nécessité d’être révisés.

Réanimation à travers des fonctions nouvelles (objectif 3). Dans le but de provoquer la réanimation et leur trouver des fonctions nouvelles correspondant à leurs volumes intérieurs et à leur décor.

- Créer des conditions plus satisfaisantes pour la vie du quartier et de ses habitants (objectif 4). Apparentement toutes les interventions ont été faites en cherchant à créer des conditions plus satisfaisantes pour la vie du quartier et de ses habitants.

\subsection{Les objectifs ajoutés depuis le PPSMV:}

La création de nouveaux objectifs à été fait à partir du PSMV (1996). Lapparition du POS (1977) a produit la mise en cohérence du PSMV avec ce document.

Les nouveaux objectifs ont été : Lassouplissement des règles de constructibilité - introduit pour satisfaire l'évolution de l'habitabilité, le confort et la sécurité - et les possibilités de constructions nouvelles. Ensuite, l'incorporation du PADD au PLU, en 2011, a produit des nouveaux objectifs au PSMV (2013) liés au développement durable et à la mixité sociale. Les nouveaux objectifs sont : maintenir la mixité sociale dans les quartiers centraux, l'intégration des objectifs du " Plan Climat », sauvegarder et développer le biotope $\mathrm{b}^{36}$.

\section{Les objectifs du PSMV (2013)}

36. «Milieu biologique déterminé offrant à une biocénose des conditions d'habitat relativement stables ». (REY Alain, Le Petit Robert, 2011)
Voir les cases de couleur orange dans le Graphique 2 Diagramme lévolution des objectifs du PSMV.

A) Renouvellement du patrimoine

La liste des bâtiments protégés a été élargie, en raison d’une notion de protection sans délimitation de période

Nouvelle classification de l'utilisation du sol : L'intégration à la liste de cette diversité de bâtiments a produit deux types de nouvelles catégories - type $\mathrm{A}^{37}$ et type $\mathrm{B}^{38}$ - caractérisées par leur intérêt patrimonial. Chacun des bâtiments a fait l'objet d'une analyse (de description et d'appréciation fondée sur la documentation historique, des visites, des reportages photographiques et des fiches d'enquête) visant à déterminer sa valeur patrimoniale.

Important croissance, de 50\% à 78\%, des bâtiments protégés : Cela est dû à l'intégration du patrimoine du XIXème siècle et moderne. Selon l'inventaire du Marais de 1970, $75 \%$ des immeubles existant à l'intérieur du périmètre du secteur sauvegardé ont été bâtis antérieurement à 1870 (Paris-Projet, 1970). Le PSMV (1996) était quant à lui concentré sur le patrimoine monumental et de l'Ancien régime, le patrimoine des XIXème et XXème siècles y étant particulièrement mal protégé (Christiane Blancot, 2004). Par conséquent, le PSMV (2013) couvre un large spectre historique du XVIème siècle jusqu'au début des années $1970^{39}$

Par ailleurs, en ce qui concerne les immeubles ou parties des immeubles de protection au titre des Monuments Historiques, on observe une baisse de $8 \%$ à $3 \%$. Cette diminution est artificielle, car dans l'ancien règlement, les protections d'un élément particulier (façade, escalier, cheminée...) avaient pour résultat l'incorporation automatique de tout le bâtiment dans le comptage des terrains protégés. (.Ibid.)

A cet égard, la proportion de bâtiments non protégés a diminué de $36 \%$ à $19 \%$.

37. Type A : Immeuble ou partie d'immeuble à conserver, dont la démolition, l'enlèvement ou l'altération sont interdits et dont la modification est soumise à des conditions spéciales.

38. Type B : Immeuble ou partie d'immeuble à conserver, dont le réaménagement, pouvant comporter des interventions sur la structure et/ou sur la répartition des volumes intérieur existants, est autorisé sous conditions.

39. « Bâtiments d'habitation, hôtels, ateliers, bâtiments d'activités, immeubles de bureaux, marchés, entrepôts, locaux commerciaux, restaurants et cafés .... [...] Leur diversité reflète la reconnaissance de nouvelles catégories de patrimoine jusqu'ici insuffisamment prises en compte : I'habitat vernaculaire caractéristique des siècles passés, l'architecture industrielle, les opérations de lotissement spéculatives du XIXème siècle, les grands courants esthétiques du XXème siècle jusqu'à la période des Trente Glorieuses.» (Atelier Blanc-Duché, PSMV de Paris le Marais - Dossier n³. Partie III Justification des règles et la compatibilité avec les autres documents d'urbanisme, Op.cit, 2013, p.28). 
La liste des bâtiments voués à la démolition a diminué, la liste de 1996 a été réalisée en fonction de l’idée initiale de raréfaction du bâti, en cohérence avec l'obligation de lutter contre l'insalubrité qu'avait provoquée la concentration d'activités industrielles depuis un siècle et la dégradation des conditions d'habitat.

Le PSMV (2013) a réalisé une révision, au cas par cas, en fonction des enjeux qui s'attachent à chaque parcelle ${ }^{40}$ afin de mieux prendre en compte les activités et conserver les différents types d'occupation des parcelles.

Changement de la surface : Le nombre de bâtiments dont la démolition peut être imposée a été divisé par presque un facteur 5 (de 14\% à 3\%). (.Ibid.).

\section{B) Diversité des fonctions urbaines}

Renforcement de la protection du commerce : Dans le PSMV (1996) le commerce n'a pas bénéficié d'une protection spécifique. Le PSMV (2013), afin d'imposer la réalisation de locaux commerciaux ou d'éviter une déstructuration du tissu commercial, propose dans le règlement, une série d’articles:

Art. US.2.2.a.2 : Les locaux situés à rez-de-chaussée sur rue doivent (en cas de construction, reconstruction ou de réhabilitation lourde) être destinés au commerce ou à l'artisanat (à l'exception des locaux d'accès aux immeubles) (Atelier Blanc-Duché, 2013).

Art. US.2.2.a.3 : Interdiction du changement de destination des locaux commerciaux ou artisanaux en rez-dechaussée sur rue (.Ibid.).

\section{C) Mixité sociale}

Augmentation du pourcentage de la surface destinée à la mixité sociale: Afin de maintenir la mixité sociale dans les quartiers centraux, l'«article US.2,3» du règlement du PSMV (2013) fixe une norme de 30\% de logement sociaux, supérieure à l'objectif de $25 \%$ fixé par la loi SRU. Ce pourcentage supérieur est destiné à marquer une volonté de rattrapage par rapport à la situation déficitaire actuelle.

\section{D) Environnement et Développement durable}

Intégration de nouvelles dispositions autorisant la mise en place de dispositif techniques: Nous avons constaté que la ville de Paris a adopté le Plan climat le $1^{\text {er }}$ octobre 2007 afin de réduire l'ensemble des émissions de gaz à effet de serre de son territoire et de ses activités propres de $75 \%$ en 2050 par rapport à 2004. (Atelier Blanc-Duché, 2013, p.29.). De cette façon le PLU a intégré de nouvelles dispositions autorisant la mise en place de dispositifs techniques sur les constructions nouvelles ou existantes, afin d'économiser

40. Locaux d'activités à protéger, patrimoine à reconnaître et protéger, typologies d'occupation des fonds de parcelles à encourager, évolution des usages hygiène et sécurité des constructions voisines. ou de faire produire de l'énergie renouvelable. « Ces actions concernent par exemple la rénovation des bâtiments, les énergies renouvelables, les déplacements, la gestion de déchets. » (Mairie de Paris, 2009, p.4.)

Réduction des possibilités de stationnement pour participer à la politique des déplacements mise en œuvre à l'échelle de Paris : Le Atelier Blanc-Duché explique, en 2013, que pour mettre en cohérence le décalage de normes entre le PSMV (1996) qui a une politique favorable à l'automobile ${ }^{41}$ et la nouvelle politique de déplacement du PADD sur :

Faire respirer Paris: une nouvelle politique des déplacements qui a pour but la limitation du stationnement, le PSMV révisé ${ }^{42}$ propose une politique de dissuasion en expliquant que « la politique de protection du patrimoine devrait de plus bénéficier de ces nouvelles dispositions car lobligation de création de places de stationnement était une contrainte forte dans un tissu urbain dense, constitué de nombreuses parcelles étroites desservies, par des voies très peu larges, avec des sous-sols souvent très riches sur le plan archéologique. Ainsi le PSMV révisé interdit toute création d'accès pour parc de stationnement en bordure des voies de moins de 8 mètres de largeur» (p.11.)

Nous voudrions ajouter d'autres aspects (traités dans la page 34 et 35 du même document) liés à cette politique de dissuasion comme :

La favorisation de l'usage des transports collectifs et la réduction de l'utilisation de la voiture en ville. Dans cette perspective, le PSMV (2013) propose de traiter différemment le stationnement lié au domicile et le stationnement lié à l'emploi.

«La possibilité d’un stationnement près du domicile doit être offerte pour permettre aux résidents de ne pas être obligés d'effectuer leurs déplacements domicile-travail en voiture. A cet effet, il faut également ne pas encourager la possibilité d'une offre stationnement près du lieu de travail, en particulier dans les secteurs très bien desservis par les transports collectifs. » (Atelier Blanc-Duché, 2013).

Lexigence d'un minimum de 2,25\% de la surface de plancher locaux (habitation et bureau) pour le stationnement des vélos et des poussettes. Cette disposition favorise le développement d'une offre privée de stationnement.

Adaptation de règles pour sauvegarder et développer le déficit de biotope: Le PADD a pour objectif de préserver le paysage urbain, améliorer la qualité et le cadre de vie

41. Pour rappel, résidait dans ce plan la nécessité d'imposer l'aménagement d'un nombre minimum de places de stationnement pour solutionner la demande de stationnement sur voirie publiques.

42. Cette source est un document préalable à l'approbation du PSMV (2013). Si bien que, le PSMV révisé fait référence au PSMV (2013). 
des habitants, sauvegarder et développer le biotope. Si bien que, les dispositions du PLU visant à créer des espaces libres supplémentaires et des cours intérieures doivent s'y appliquer. Cependant, nous avons constaté que la plus grande part des espaces non bâtis du Marais sont constituée de cours pavées, c'est-à-dire que seuls les hôtels particuliers importants possèdent un jardin à l'arrière du corps principal d'habitation. Il y a 9,9 ha. de cour (ce qui équivaut à $55 \%$ ) et 8,3 ha. de jardin (équivalent à $45 \%$ ). Les calculs montrent que le Marais constitue un secteur de déficit de biotope. Il existe seulement 8\% d'espace libre dédié au domaine végétal par rapport à la surface totale des parcelles. Ainsi, pour éviter une possible contradiction avec les prescriptions patrimoniales, les règles ont été adaptées.

\section{Conclusion}

En 1969, a été réalisé le premier projet, le PPSMV. Etant donné que le Marais est devenu à ce moment un grand centre de la fabrique et du négoce, un des quartiers le plus vétuste, le plus insalubre, le plus encombré et le moins aéré de la capitale ; le PPSMV, avec une idée de modernisation, a proposé un "Plan de curetage " pour faire disparaitre ces problèmes d'hygiène et salubrité. Il propose la récupération des îlots et des cours des immeubles encombrés de construction pour les transformer en espaces libres d'aménagement végétal, indispensables à l'aération.

Dans notre analyse, nous avons identifié que le deuxième PSMV (1976) et le troisième PSMV (1980) ont correspondu plutôt à un réajustement des objectifs faits au PPSMV (1969). Il n'avait pas de création de nouveaux objectifs. Donc, nous pouvons parler d'une mise à jour de ses objectifs initiaux.

Le quatrième PSMV (1996), est le résultat de un ajustement de la sauvegarde du patrimoine, qui a mis à jour documents graphiques et amélioré la rédaction du règlement, il a fait un règlement qui se rapproche au mieux du POS (1977) de Paris. Dans cette perspective, nous avons vu la création de nouveaux objectifs. Il a eu un intérêt d'assouplissement des règles de constructibilité pour satisfaire l'évolution de l'habitabilité, le confort et la sécurité ; et un intérêt pour la possibilité de constructions nouvelles.

Ensuite, en 2000, la loi SRU a modifié en profondeur le droit de l'urbanisme et du logement en France, un des principaux impacts a été le remplacement des POS par le PLU, plus planification et moins réglementaire. Le PSMV de 1996 n’envisage pas certaines dispositions du PLU liés au PADD et au Plan Climat. Dans cette perspective, afin d'éviter contradictions entre le décalage des orientations fixées, le PSMV (2013) met en cohérence le PSMV (1996) avec les nouveaux dispositions issue du PLU. Nous avons constaté aussi que le PSMV (2013) prend de nouvelles notions liées à une problématique mondiale, comme les gaz à effet de serre. Certains plans, lois et règlements ont été déjà imposés. L’expérience nous montre que nous donnons de l'importance, ou autrement dit «mettons en valeur» ce qui est au risque de disparaitre ou qui peuvent impliquer de conséquence désastreuse. A cet égard, nous avons observé qu'aujourd'hui nous parlons aussi de la mise en valeur du patrimoine végétal. C'est l'insertion des notions comme : développement durable, écologie, environnement... Au début, le PPSMV (1969) proposait le «plan de curetage», un projet qui proposait de reconquérir progressivement dans le cœur des îlots ou dans la cours des immeubles la récupération de l'utilisation du sol destinée à l'aménagement végétal. C'était l'amélioration de la qualité de vie à travers l'aération de la ville. Cependant, aujourd'hui le PSMV (2013) prend les dispositions du PADD et du PLU pour améliorer durablement le cadre de vie quotidien de tous les parisiens ; promouvoir le rayonnement de Paris et stimuler la création d'emplois pour tous ; impliquer tous les partenaires, acteurs de la mise en œuvre du projet ; et réduire les inégalités pour un Paris solidaire et le Plan Climat -actions pour diminuer l'émission de gaz à effet de serre- . Donc, le règlement propose l'intégration de nouvelles dispositions autorisant la mise en place des dispositifs techniques qui doivent économiser l'énergie ou produire de lénergie renouvelable dans les constructions. Ces actions concernent par exemple la rénovation des bâtiments, les énergies renouvelables, les déplacements, la gestion de déchets, etc.

En substance, nous avons constaté que le projet du PSMV a été le résultat d'un processus lent de définition et d'adaptations successives. À partir du bilan de la littérature nous pouvons affirmer que le PSMV (2013) a élaboré des rectifications légales de grande efficacité. Les processus de révisions et les études permanentes ont permis de produire un document qui surmonte les dispositions de contradictions apparu depuis de certains années, il rendre le projet un document qui marche et qui s'adapte aux besoins réels de ce qui l'utilisent. Ainsi, le PSMV (2013) n'a pas seulement bien expliqué la compatibilité avec d'autres documents d'urbanisme et la justification des nouvelles règles, mais il a aussi mis à jour les anciens objectifs et apporté des nouveaux. Il a connu de profondes évolutions au cours de ces dernières années, tant du fait de la politique urbaine parisienne que de la politique patrimoniale de l'Etat. Plus que sur les aspects de la protection, ce sont donc aujourd'hui sur ceux de la mise en valeur du patrimoine que portent les grands enjeux du PSMV. Nous avons vu que le regard sur le patrimoine s'est également transformé. Au début les monuments historiques et les secteurs sauvegardés étaient perçus sous le seul angle patrimonial. Parallèlement les documents de planification urbaine ont également évolué dans leurs préoccupations. Aujourd'hui, le PSMV porte une vision plus hybride intégrant à la fois la sauvegarde du patrimoine et la planification urbaine. Sa convergence avec les documents de planification urbaine règlementairement obligatoire tend à sa fusion avec ceux-ci. 